\title{
Movilidad, espacio y diseño: metodología de análisis integrada del sistema viario en relación a sus contextos y a parámetros de diseño. El caso de la ciudad de la Plata
}

\author{
Mobility, space and design: A methodology to analyze the road system in relation to its context and design parameters. \\ Case study: La Plata, Argentina
}

María Julieta López* y Olga Rosa Ravella*

Recibido: 06 de septiembre de 2018

Aceptado: 22 de abril de 2019

\section{Resumen}

En la última década los espacios del sistema de movilidad están siendo analizados desde nuevos enfoques. Enfoques que integran aproximaciones teóricas de diversas disciplinas y que avanzan sobre la tradicional concepción ingenieril de las infraestructuras. En línea con estos nuevos estudios, se expone una metodología que vincula dos escalas de análisis, la primera contextualiza al sistema viario para entender su rol en el sistema territorial que integra y la segunda detalla sus propiedades aplicando un sistema de parámetros de diseño, con el fin de comprender su incidencia en la movilidad, accesibilidad y habitabilidad urbana. El artículo propone como caso de aplicación para dicha metodología a la ciudad de La Plata, a partir de considerarla un caso representativo del conjunto de ciudades intermedias argentinas. Dichas ciudades han experimentado cambios importantes en su movilidad producto de la expansión urbana de las últimas décadas, sin el respaldo de instrumentos de gestión que contemplaran al sistema vial de manera integrada. Se presenta un repaso de la bibliografía más importante en materia de análisis y diseño urbano, profundizando detalladamente en la metodología propuesta, y evidenciando los resultados del caso. Se expone sintéticamente reflexiones sobre las escalas analíticas y los criterios clave que deben favorecer al diseño y organización del sistema viario.

Palabras clave: diseño urbano, espacio, movilidad, parámetros de diseño.

\begin{abstract}
In the last decade the spaces of the mobility system are being analyzed from new approaches. Approaches that integrate theoretical approaches of different disciplines and that advance on the traditional engineering conception of infrastructures. In line with these new studies, a methodology linking two scales of analysis is presented, the first contextualizes the road system to understand its role in the territorial system it integrates and the second details its properties by applying a system of design parameters, with the in order to understand its impact on mobility, accessibility and urban habitability. The work proposes as a case of application for this methodology to the city of La Plata, from considering it a representative case of the set of intermediate Argentine cities. These cities have experienced significant changes in their mobility as a result of the urban expansion of recent decades, without the backing of management instruments that contemplate the road system in an integrated manner. The communication presents a review of the most important literature on urban analysis and design, delves deeply into the proposed methodology, evidences the results of the case and synthetically presents reflections on the analytical scales and the key criteria that should favor the design and organization of the road system Keywords: design parameters, mobility, space, urban design.
\end{abstract}

* Filiación: Instituto de investigaciones y política del ambiente construido. Facultad de Arquitectura y Urbanismo. Universidad Nacional de La Plata, La Plata, Argentina. Contacto arq.julietalopez@gmail.com y oravella@gmail.com

El presente artículo junto con su metodología es parte de una reelaboración de la tesis Red viaria, ciudad y paisaje. Aproximación teórica metodológica para su diseño de María Julieta López, 2016.

Cómo citar: López, M. J. y Ravella, O. (2019). Movilidad, espacio y diseño: metodología de análisis integrada del sistema viario en relación a sus contextos y a parámetros de diseño: el caso de la ciudad de la plata. Revista de Urbanismo, 40, 1-25. https://doi.org/10.5354/0717-5051.2018.51111 


\section{Introducción}

\section{La ciudad y el diseño del sistema espacial de movilidad}

La discusión de este artículo se ancla en el cruce de dos temas de gran complejidad: (i) la movilidad urbana generada por el crecimiento de las ciudades argentinas y (ii) el diseño particular que se le ha dado al sistema viario por el cual subyace una concepción funcional de este elemento.

La concepción espacial de las ciudades argentinas, en su mayoría, fue en base a un plano en cuadricula, asociado a una red viaria compuesta por avenidas principales y calles secundarias. A lo largo del proceso histórico de su evolución, comenzaron a presentar desbordes por el crecimiento poblacional (UTDT-CIPUV, 2013). Estos desbordes, en su mayoría, han sido guiados por la lógica del mercado de suelo por sobre los instrumentos de planificación, produciendo sistemáticamente áreas residenciales de baja densidad estructuradas a los primeros caminos o vías interurbanas de acceso a la ciudad (Ravella, 2000; Janoschka, 2002; Frediani, 2010; Aón, Giglio y Cola, 2017; Freaza, Giglio y Aón, 2017).

Esta dinámica de crecimiento urbano, a diferencia de las ciudades planificadas en las que la urbanización del suelo rural precede al asentamiento de la población, se caracteriza por la ocupación del suelo en primer lugar y luego una progresiva urbanización, hecho que explica las notables diferencias de pautas sociales, culturales e institucionales sobre las que se asienta la producción del territorio urbano en ambos casos (Ministerio de Planificación Federal, 2011).

En relación con la movilidad, la lógica de crecimiento de las ciudades intermedias argentinas es el desarrollo, primordialmente, sobre los ejes de acceso y redes principales del sistema viario, sin contemplar la distribución de los equipamientos, generando de esta manera una elevada dependencia entre estos sectores de crecimientos y las áreas centrales de las ciudades, donde se ubican los centros administrativos, escuelas, hospitales y corredores comerciales. Esta variable de dependencia condiciona proporcionalmente el aumento de movilidad urbana y presión sobre la red viaria existente, fundamentalmente por la necesidad de medios motores (auto, moto, transporte público) al incrementarse las distancias.
La triple combinación de aumento de viajes, aumento de distancias y aumento del uso de medios motorizados, produce un aumento del tránsito, de la siniestralidad vial y de la contaminación ambiental; colapsando de esta manera las infraestructuras que dan soporte a la movilidad y convirtiendo la accesibilidad en una situación crítica para los ciudadanos.

En Argentina, al comparar los sistemas viarios de la mayoría de las ciudades, se destaca un tipo de gestión espacial que sigue una lógica funcional y, a pesar la existencia de una importante base bibliográfica sobre el análisis y proyecto del sistema viario, son escasos las plataformas de diseño que coadyuvan a crear instrumentos para repensar su concepción. Es en este punto, que esta comunicación plantea su aporte: exponer una metodología de análisis del espacio viario que posibilite no solo describir la realidad en estudio, sino que constituya un instrumento de visibilización de temasproblemas claves para la toma de decisiones.

La escasez de plataformas de diseño integradas se explica, en parte, por la resistencia de las disciplinas encargadas de proyectar y gestionar el espacio viario a asumir una posición complementaria, no exclusiva, para la resignificación, estudio y proyección del sistema viario. Haciendo una revisión histórica se puede observar que esta situación se vislumbraba desde el siglo XIX, a partir de la teoría general de la urbanización (1867) de Ildefonso Cerdá (España, 1815 - 1876), donde se anunciaba un primer orden teórico que contenía, por un lado, a las vías: como el lugar donde se desarrolla la vialidad y, por otro lado, a las intervías: refiriéndose a las manzanas. Esta concepción, movilidad y estancia, favorecía un enfoque vinculante, que no se dirigía solo al estudio de los edificios o solo al estudio de la vía. Como señala Soria y Puig (1999), al contemplar:

[V]ías e intervías como un par inseparable, se trataba de evitar dos peligros siempre latentes: que los ingenieros diseñen vías sin tener clara conciencia de que con ello definen variables fundamentales para la habitabilidad de las intervías, o que los arquitectos diseñen manzanas o intervías por simple agregación de edificios sin pensar en las características funcionales de la red viaria resultante. (Soria y Puig, 1999, p. 33) 
Un siglo más tarde con la creación del auto, este peligro latente, terminó siendo el tema más crítico: la dificultad de construir un marco teórico-metodológico de diseño entre las disciplinas encargadas de planificar y gestionar el espacio de la movilidad que, y que en los últimos años comenzó lentamente a revertirse. La separación de las diferentes disciplinas encargadas de teorizar e intervenir en el sistema viario, se refleja principalmente por el avance de diversas corrientes de pensamiento. Por una parte, las vías abordadas desde la perspectiva de la ingeniería, en el marco del racionalismo. Por otra parte, la perspectiva que aborda la relación entre dichas vías desde las diversas características del transporte, así como también desde la perspectiva perceptual y paisajística y, por último, el viario como sistema y elemento componente del espacio público de la ciudad.

\section{El viario: función, espacio y forma}

En este grupo se destacan conocimientos e investigaciones que han irrumpido en la temática confiriendo nuevas formas de concebir al espacio de la movilidad a partir de incorporar multiplicidad de escalas y avanzar en el diseño fusionando parámetros geométricos y de función.

Al introducirnos en prototipos de redes viarias, un modelo que fue evolucionando es la greenway o vía parque (1980) de Frederick Law Olmsted (Estados Unidos, 1822-1903) y Calvert Vaux (Londres, 1824-1895) cuyas recomendaciones contribuyeron a la mejora del sistema viario. Continuando con el enfoque funcional-formal: Charles Édouard Jeanneret (Suiza, 1887-1965) (en adelante, Le Corbusier) ya en el año 1924, planteaba la necesidad de articulación de la red viaria mediante la diferenciación de los distintos tipos de transporte, lo que condujo más adelante al origen de la teoría de las 7 vías, que fue materializada en la ciudad Chandigarh (1951) (López, 2016, p.32).

Los planteos de Le Corbusier apoyados en y por el Congreso Internacional de Arquitectura Moderna (CIAM) (1928-1959), suponen los primeros intentos de clarificación de la naturaleza y el significado arquitectónico-funcional de las redes viarias, la necesidad de clasificación según tipologías/jerarquías y del análisis integral del transporte urbano (López, 2016, p.33).
Por otro lado, desde otro enfoque y ampliando las escalas de análisis Colín Buchanan (India, 1907-2001) con su obra The traffic in towns (1963), exponía las relaciones tan fuertes que se daban entre tráfico, desarrollo urbano y vías, sus consecuencias a largo plazo, así como su incidencia en el ambiente humano. Como indica Fariña en su blog (2012):

Buchanan planteaba algunas cuestiones que hoy parecen casi tópicas: la frustración producida por el uso del coche; los accidentes y la siniestralidad; el deterioro producido en el ambiente por ruidos, humos, olores, intrusiones visuales, estrés, ansiedad y problemas de salud debidos a la contaminación; y otras, como el sprawl urbano que aparece como un problema.

Afirmaba que la planificación de una vía debía ser pensada en relación con los usos que se acontecían porque eran las actividades las que generaban diferentes tipos de atracción o generación de viajes. En este sentido, Buchanan volvía al enfoque de interdependencia para entender la dinámica del tránsito en las ciudades.

El diseño de las vías se comenzó a guiar en base a los enfoques de la Ingeniería de tránsito. Fueron cada vez más hegemónicos los procedimientos técnicos basados en datos tomados en campo sobre las características del tránsito, las vías y la capacidad de estas. A partir de 1950 cuando se construye el primer Manual de Capacidad Vial (Highway Capacity Manual) las investigaciones dieron un salto buscando modelizar patrones de movilidad que suponían predecir demandas futuras y con esos datos definir áreas críticas que reclamarían una solución.

Como expresa en su tesis el ingeniero en caminos Herce (2009), este enfoque da un aparente sustento científico al dimensionado de las redes, mediante la aplicación de sofisticados métodos de cálculos que proporcionaban a la planificación una presunta autonomía, respecto de otras consideraciones propias del planeamiento urbanístico. Esta conceptualización especializada, impuesta en la concepción de las redes aproximadamente desde el año 1940, supuso que cada una de las infraestructuras fuera concebida tan sólo desde sus propios requerimientos de funcionalidad y de demanda de consumo. Por ello, los manuales de dimensionado de cualquiera de las 
redes de infraestructuras - no solo de transporte parten en general de variables tales como: caudal - presión - velocidad - intensidad y sobre estos indicadores se plantean la organización de la red, su extensión, dimensión y capacidad (López, 2016, p.35).

\section{El viario desde la imagen, percepción, la escala humana}

En los años 60 se comienza a manifestar ciertas críticas sobre el funcionamiento y las concepciones de las infraestructuras viaria. Buscando nuevas formas de abordar a este elemento se empezaron a desarrollar análisis en pequeña escala, significando los entramados peatonales y profundizando en el conocimiento de la humanización de los espacios. Dos autores importantes de este grupo fueron Gordon Cullen (Inglaterra, 1914 - 1994) con su obra El paisaje urbano (1959) y Sylvia Crowe (Inglaterra, 1901-1997) con Landscape of road (1960).

En la misma línea, pero diferenciándose en la metodología, abordando una visión del conjunto que no haga a la vía único objeto de análisis, comienzan a reconocerse diversos aporte, entre los cuales se destacan los trabajos de Kevin Lynch (Estados Unidos, 1918-1984): La imagen de la ciudad (1960), Site Planning (1962), The View from the Road (1964) y Growing Up in Cities (1977) y los estudios de Lawrence Halprin (Estados Unidos, 19162009) Freeways (1966) y Geoffrey Jellicoe (Reino Unido, 1900-1996) Studies in Landscape design (1966). El desarrollo de estos enfoques determina la apertura de los estudios desde la psicología ambiental en ámbitos urbanos, que avanzan en el conocimiento de la valoración y experiencia del espacio, explicando técnicamente la relación entre el comportamiento y el medio ambiente (López, 2016, p.38).

Los avances del primer grupo tienen tal valoración metodológica y técnica, que hasta la actualidad continúan desarrollándose. Pero, comienzan a visibilizarse sus incongruencias como, por ejemplo, que sus propuestas conciben al mundo como un lugar ilimitado a nivel espacial donde solo la técnica puede dar garantía de satisfacción. Sin embargo, en la mayoría de las ciudades argentinas, este tipo de enfoque y propuestas escapan de los problemas reales de los ciudadanos.

\section{El viario como sistema soporte de la movilidad y factor organizativo del espacio público de la ciudad}

Frente a la crisis mundial ambiental y a las nuevas sociedades que giran en base a las nuevas tecnologías, nuevos interrogantes comenzaron a realizarse sobre la movilidad, la ciudad, las infraestructuras, el desarrollo de estas. Este cambio de paradigma da cuenta de que transporte y movilidad no son sinónimos y que al ubicar al sector transporte como consumidor de energía y a la movilidad como concepto que engloba a todas las formas de desplazamiento, el espacio viario toma nuevas perspectivas, más amplias.

Los avances de diversas investigaciones (Arroyo, 1992; Manchón y Santamera, 1995; Pozueta Echavarri, 2014; NACTO, 2018; Herce, 2009) conciben al sistema espacial de la movilidad como factor de accesibilidad y esto reivindica que todas las formas de desplazamiento tienen importancia en el sistema y que la inexistencia de espacios adaptados a los requerimientos de las formas más autónomas de desplazamiento supone una marginación de este tipo de movilidad, imposibilitando la conexión a amplias capas de población. Como demuestra Herce esta perspectiva está reflejando un cambio respecto al modo de enfocar la planificación y gestión de la movilidad urbana, y tal como muestra el autor, este cambio se caracteriza por la búsqueda de instrumentos de atención al ciudadano a muy distintos niveles (López, 2016, p.52).

Bajo esta línea, el diseño urbano se pone en valor, ya que se constituye una herramienta para pensar y readaptar el espacio de la ciudad a estas exigencias y se da valor agregado a la importancia de construir o definir parámetros de diseño, que son más que condiciones geométricas (Dupuy, 1995; Herce, 2009; Naredo y Rueda, 1996)

Un ejemplo es NACTO, una organización que busca mejorar el transporte, público o privado, y el diseño de las calles para que éstos se enfoquen en las necesidades reales de las personas. Se presentan herramientas y técnicas para hacer ciudades con calles más habitables, seguras y más atractivas en términos comerciales.

En correspondencia teórica a estos últimos avances se construye la metodología que seguidamente se detallara. En particular interesa destacar que la estructura metodológica propuesta se organiza en base a dos escalas de análisis para comprender y caracterizar el sistema 
viario como subsistema vinculante a las particularidades de la ciudad y por otro, valorar en detalle la calidad de la infraestructura a partir de la elaboración de un inventario que se conforma por un sistema de parámetros de diseño.

El inventario se conforma por 6 parámetros que vinculan un conjunto ordenado de 23 variables, cuyo objetivo es proveer de una visión totalizadora respecto a la realidad observada, intentando definir i) los aspectos que posibilitan la desvinculación de las redes viarias; ii) los elementos que se relacionan con su acondicionamiento; iii) los parámetros clave de diseño para aumentar su habitabilidad

Es claro que el tema permite tantas respuestas como compleja es nuestra realidad urbana, sin embargo, es posible mediante el avance de estas metodologías, no solo describir la realidad en estudio, sino también establecer estándares que permitan luego determinar perfiles de calidad de una vía, o de un sistema de vías, en una determinada ciudad. El aporte se traduce en contribuir a una serie de instrumentos de apoyo a la hora de tomar decisiones.

Para la validación de resultados se seleccionó como caso de estudio la ciudad de La Plata ${ }^{1}$ ya que representa al conjunto de ciudades intermedias argentinas, que han experimentado importantes cambios morfológicos, sociales y han tenido giros importantes en sus patrones de movilidad debido a la falta de instrumentos de gestión del sistema vial ocasionando externalidades negativas: contaminación del aire, siniestralidad vial y congestión.

La comunicación se estructura en tres apartados principales. La primera parte presenta y detalla rigurosamente la estrategia metodológica de análisis del espacio de la movilidad. La segunda parte se exponen los resultados obtenidos en el caso seleccionado: la ciudad de La Plata y, por último, se reflexiona y concluye sobre las escalas analíticas y los criterios clave que deben favorecer al diseño y organización del sistema viario

\section{Propuesta teórica - metodológica}

El punto de partida para el desarrollo de la propuesta metodológica fue, por un lado, la integración de los conceptos de transporte y movilidad, ciudad y territorio, como componentes de un sistema complejo emergente de la relación sociedad-naturaleza y, por otro, la comprensión de la red viaria como un elemento estructurante del desarrollo físico-espacial urbano que incide por su estructura y diseño en el aumento/reducción de la movilidad y accesibilidad. Por tanto, el primer criterio metodológico que se plantea para el abordaje de la red viaria es la necesidad de entenderla de manera contextualizada en su historia y como parte del sistema soporte de un ámbito territorial específico.

El segundo criterio metodológico, por otro lado, parte de considerar a la red viaria como un elemento significativo de los espacios públicos, como un componente básico de organización de la trama urbana que, según su estructura, materialidad y usos, influirá en la movilidad, accesibilidad y habitabilidad. A partir de
${ }^{1}$ La Plata es capital de la provincia de Buenos Aires. Se ubica a $56 \mathrm{~km}$ al sudeste de la ciudad de Buenos Aires. Es la $4^{\circ}$ ciudad más poblada del país y el $5^{\circ}$ aglomerado urbano con más habitantes después de Buenos Aires, Córdoba, Rosario y Mendoza. Es apodada frecuentemente como dicho enfoque, el segundo criterio metodológico plantea la necesidad de entender detalladamente la composición y elementos constituyentes de la vía, construyendo un inventario compuesto por un sistema de parámetros de diseño.

En correspondencia a dicho planteo inicial, el procedimiento metodológico se estructura en dos niveles de análisis vinculantes entre sí: el nivel superior y el nivel de anclaje (Samaja, 1995)

Desde el nivel superior. (Figura 1) Se caracteriza la ciudad y el espacio viario mediante dos aproximaciones:

a. La histórica: implica analizar a través de cortes históricos las siguientes variables: i) las formas de desarrollo urbano, ii) los principios estructurantes del crecimiento; iii) la simbología que representa las infraestructuras de movilidad y iv) la evolución de los patrones de movilidad de la ciudad.

b. La urbana territorial: implica reconocer los rasgos principales del sistema construido y social: población,

la «Ciudad de las Diagonales» y en menor medida como la «Ciudad de los Tilos» (López, 2016, p.22). 
densidad, hogares, cantidad de viviendas, NBI, patrones de movilidad, usos de suelo; vinculándolos al sistema espacial viario jerarquizado.

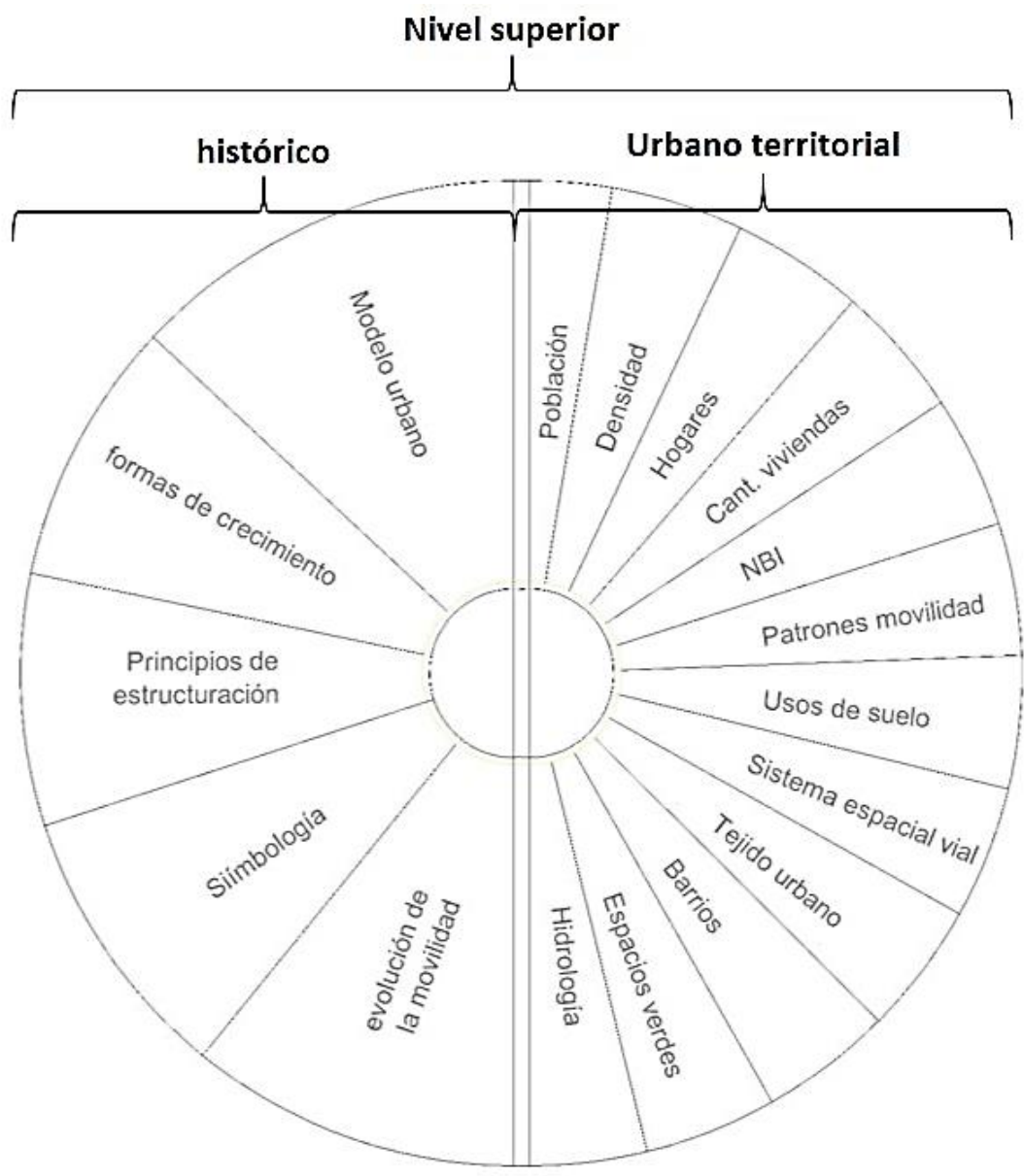

Figura 1. Nivel anclaje: parámetros de diseño y sus variables. Fuente: Elaboración propia, 2018.

Desde el nivel de anclaje. ( Figura 2) se continúa con el análisis viario mediante la construcción de un inventario fusionando los siguientes seis parámetros de diseño determinados en el marco de la movilidad sustentable y tomando la dimensión social, ambiental, funcional y paisajística que, desde este trabajo, se consideran clave para orientar el proceso de diseño urbano2: 1) Vinculación socio espacial; 2) Espacial geométrico; 3) Integración funcional; 4) Habitabilidad; 5) Seguridad y 6) Potencial paisajístico (López, 2016, p.62).

Cada parámetro es producto de la fusión de varias dimensiones, en total son 23 variables que implican la utilización de datos empíricos expresados en unidades heterogéneas, siendo imposible, en dicho estado, su integración. Por tanto, se precisó su transformación en otro tipo de unidad homogénea directamente integrables.
${ }^{2}$ Movilidad sustentable: Condiciones en relación a lo: i) Social: la vinculación entre los ciudadanos, los barrios y la ciudad; ii) Funcional: integración entre tipos de vía, su función y el contexto socio espacial que atraviesa; iii) Ambiental: acondicionamiento mínimo de confort y seguridad que el espacio debiera presentar; iv) Paisajístico: generar referencialidad del espacio urbano y las redes viarias, mediante legibilidad y calidad visual (López, 2016, p.62). 
Para ello, se siguió el método estándar de los puntos de correspondencia3 ${ }^{3}$ Leva, 2012)

Una vez unificada la métrica, mostrará un valor entre 0 y 100 puntos, mientras más alto es el puntaje se asume como mejor la calidad de la vía en su totalidad y simultáneamente permite reconocer la comparación entre las variables particulares. En correspondencia para visualizarlo mejor por cada ponderación se determina un color.

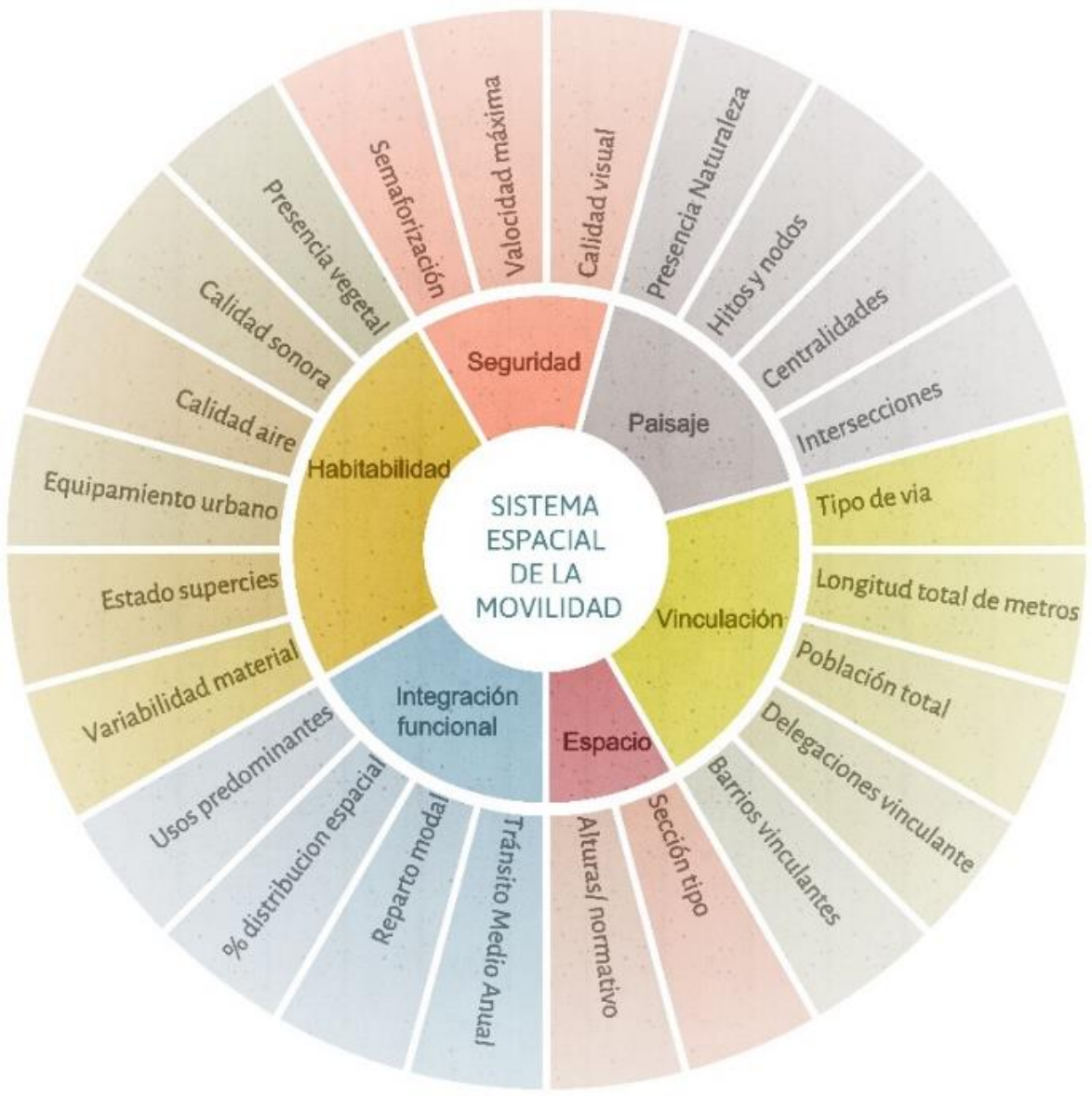

Figura 2. Nivel anclaje: parámetros de diseño y sus variables. Fuente: Elaboración propia, 2018.

1. El parámetro vinculación socio espacial. Este parámetro da cuenta del grado de conexión del viario entre la población, los barrios y la ciudad. Tiene una valoración de 20/100 al interior del conjunto del inventario. Como se muestra en la Tabla 1 se plantea las siguientes variables: i) Tipo de vía según normativa y flujos realizados por conteos; ii) Longitud de la vía total en $\mathrm{km}$; iii) La población directa/ indirecta en cantidad y en relación porcentual al total de población de la ciudad que sirve según zona de crecimiento que atraviesa; iv) el número de delegaciones y barrios vinculados directamente a la vía.

${ }^{3}$ Para mejor detalle ver Leva, 2012 


\section{Tabla 1}

Valores y variables del parámetro vinculación socio espacial

\begin{tabular}{|c|c|c|c|c|}
\hline PARÁMETRO & VARIABLES & Ponderación & Rangos & $\mathbf{V}$ \\
\hline \multirow{8}{*}{$\begin{array}{c}\text { VINCULACIÓN SOCIO } \\
\text { ESPACIAL } 20\end{array}$} & \multirow{5}{*}{$\begin{array}{c}\text { Tipo de vía según flujos } \\
\text { conteos y jerarquía } \\
\text { normada }\end{array}$} & \multirow{5}{*}{4} & Primer nivel urbana regional & 4 \\
\hline & & & segundo nivel urbana regional & 3 \\
\hline & & & primer nivel urbana & 3 \\
\hline & & & segundo nivel urbana & 2 \\
\hline & & & tercer nivel urbana & 1 \\
\hline & \multirow{3}{*}{$\begin{array}{l}\text { Longitud de la vía continua } \\
\text { total en } \mathrm{km}\end{array}$} & \multirow{3}{*}{3} & alto (> $15 \mathrm{~km})$ & 3 \\
\hline & & & entre (15 km y 1 km) & 2 \\
\hline & & & $(<1 \mathrm{~km})$ & 1 \\
\hline \multirow{9}{*}{$\begin{array}{c}\text { > a } 10 \text { Alta (verde) } \\
\text { e } 10 \text { a } 5 \text { Media (amarilla) } \\
\text { e } 5 \text { a } 0 \text { baja (rojo) }\end{array}$} & \multirow{3}{*}{$\begin{array}{l}\text { Población que ofrece } \\
\text { conectividad \% del total de } \\
\text { la ciudad (censo 2010) }\end{array}$} & \multirow{3}{*}{6} & Alto (> $40 \%$ población) & 6 \\
\hline & & & Medio (20\% a 39\% población) & 5 \\
\hline & & & Bajo (<20\% de población) & 2 \\
\hline & \multirow{3}{*}{$\begin{array}{c}\text { Nro. de delegaciones que } \\
\text { conecta } \% \text { del total }\end{array}$} & \multirow{3}{*}{4} & Alto (>40\% población) & 4 \\
\hline & & & Medio (20\% a 39\% población) & 2 \\
\hline & & & Bajo (<20\% de población) & 1 \\
\hline & \multirow{3}{*}{$\begin{array}{c}\text { Nro. barrios } \\
\text { atravesados \% del total }\end{array}$} & \multirow{3}{*}{3} & Alto (> 40\% población) & 3 \\
\hline & & & Medio (20\% a 39\% población) & 2 \\
\hline & & & Bajo (<20\% de población) & 1 \\
\hline
\end{tabular}

Fuente: Elaboración propia, 2018.

2. El parámetro espacial geométrico. Determina el grado de flexibilidad o de posibilidad de cambio de una vía a partir del análisis y ponderar el perfil de la calle existente, el normado por ordenanza, y el tipo de espacialidad. Tiene una valoración de 15/100 al interior del conjunto del inventario (Tabla 2).

Tabla 2

Valores y variables del parámetro espacial geométrico

\begin{tabular}{|c|c|c|c|c|}
\hline PARÁMETRO & VARIABLES & Ponderación & INDICADORES & $\mathbf{V}$ \\
\hline \multirow{3}{*}{$\begin{array}{c}\text { ESPACIAL } \\
\text { GEOMÉTRICO } \\
15 \%\end{array}$} & \multirow{3}{*}{ Sección tipo } & 10 & $>20$ m no consolidado & 10 \\
\hline & & & e/ $19 \mathrm{~m}$ a $10 \mathrm{~m}$ no consolidado & 5 \\
\hline & & & $<10$ m consolidado & 1 \\
\hline \multirow{3}{*}{$\begin{array}{c}\text { > a } 15 \text { Alta - Verde } \\
\text { e } 14 \text { a } 5 \text { Media - } \\
\text { Amarillo } \\
\text { e } 4 \text { a } 0 \text { baja - rojo }\end{array}$} & \multirow{3}{*}{$\begin{array}{c}\text { Alturas reales / } \\
\text { normativa }\end{array}$} & 5 & Perfil densificado +4 niveles & 5 \\
\hline & & & perfil medio hasta 3 niveles & 3 \\
\hline & & & bajo 1 nivel & 1 \\
\hline
\end{tabular}

Fuente: Elaboración propia, 2018. 
3. El parámetro integración funcional. Este parámetro refleja el nivel de integración entre la vía, su funcionalidad y el contexto que atraviesa. Tiene una valoración de 20/100 al interior del conjunto del inventario. Se determina según i) flujo de movilidad ii)
Identidad usuario según encuesta Origen-Destino iii) Porcentaje del reparto espacial para cada medio de transporte; iv) Perfil de usos predominantes, atracción de corredor respecto a la ciudad (Tabla 3 ).

Tabla 3

Valores y variables del parámetro integración funcional

\begin{tabular}{|c|c|c|c|c|}
\hline PARÁMETRO & VARIABLES & ponderación & INDICADORES & V \\
\hline \multirow{8}{*}{$\begin{array}{l}\text { INTEGRACIÓN FUNCIONAL } \\
20 \%\end{array}$} & \multirow{3}{*}{$\begin{array}{l}\text { Transito Medio Diario Anual 2008- } \\
2011 \text { Proyección TMDA a } 2020\end{array}$} & \multirow{3}{*}{5} & Alto $(+20.000)$ & 5 \\
\hline & & & Medio (+4000) & 3 \\
\hline & & & Bajo (+2000) & 1 \\
\hline & \multirow{4}{*}{ Encuesta Origen Destino } & \multirow{4}{*}{5} & Perfil mixto & 5 \\
\hline & & & Perfil público por sobre privado & 3 \\
\hline & & & Perfil privado por sobre público & 2 \\
\hline & & & privado & 1 \\
\hline & & & Alto (>40\%) & 3 \\
\hline \multirow{8}{*}{$\begin{array}{l}\text { > a } 15 \text { Alta } \\
\text { e } 14 \text { a } 5 \text { Media } \\
\text { e } 5 \text { a } 0 \text { baja }\end{array}$} & Numero de centralidades \% del total & 3 & Medio (20\% a 39\%) & 2 \\
\hline & & & Bajo $(<20 \%)$ & 1 \\
\hline & \multirow{3}{*}{$\%$ de reparto espacial modal } & \multirow{3}{*}{5} & Eficiente $\% \mathrm{~m}^{2}$ (>40\% reparto modal) & 5 \\
\hline & & & Admisible (>40\% $\mathrm{m}^{2}$ motor) & 3 \\
\hline & & & Deficiente (<41\% $\mathrm{m}^{2}$ motor) & 1 \\
\hline & \multirow{3}{*}{ Perfil usos predominante } & \multirow{3}{*}{3} & Comercial & 3 \\
\hline & & & Mixto & 2 \\
\hline & & & Residencial & 1 \\
\hline
\end{tabular}

Fuente: Elaboración propia, 2018. 
4. El parámetro habitabilidad. Tiene una valoración de 25/100 al considerar las condiciones que confieren confort al espacio público de la vía. Se considera: i) variabilidad de superficies ii) estado iii) equipamiento iv) calidad sonora v) presencia elemento vegetal (Tabla 4).

\section{Tabla 4}

Valores y variables del parámetro habitabilidad

\begin{tabular}{|c|c|c|c|c|}
\hline PARÁMETRO & VARIABLES & ponderación & INDICADORES & V \\
\hline \multirow{7}{*}{$\begin{array}{c}\text { HABITABILIDAD } \\
25 \%\end{array}$} & \multirow{3}{*}{ Superficies: variabilidad material } & \multirow{3}{*}{3} & eficiente (>75\%) & 3 \\
\hline & & & admisible (50\% a $74 \%)$ & 2 \\
\hline & & & deficiente $(<25 \%)$ & 1 \\
\hline & \multirow{3}{*}{ Superficies: estado } & \multirow{3}{*}{2} & eficiente (>75\%) & 2 \\
\hline & & & admisible (50\% a $74 \%$ ) & 1 \\
\hline & & & deficiente $(<25 \%)$ & 0.1 \\
\hline & \multirow{3}{*}{$\begin{array}{l}\text { Valoración Cant. y estado de } \\
\text { equipamiento }\end{array}$} & \multirow{3}{*}{5} & eficiente (>75\%) & 2 \\
\hline \multirow{8}{*}{$\begin{array}{l}\text { > a } 10 \text { Alta } \\
\text { e } 9 \text { a } 5 \text { Media } \\
\text { e } 5 \text { a } 0 \text { baja }\end{array}$} & & & admisible (50\% a $74 \%$ ) & 1 \\
\hline & & & deficiente $(<25 \%)$ & 0.1 \\
\hline & \multirow{3}{*}{$\begin{array}{c}\text { calidad sonora ( } \mathrm{dB} / \text { inferencia } \\
\text { TMDA) }\end{array}$} & \multirow{3}{*}{5} & $<40 \mathrm{~dB}$ & 5 \\
\hline & & & e $41 d B$ a $65 \mathrm{~dB}$ & 3 \\
\hline & & & $>65 \mathrm{~dB}$ & 1 \\
\hline & \multirow{3}{*}{$\begin{array}{l}\text { \% presencia elemento vegetal } \\
\text { (alta - media-baja) }\end{array}$} & \multirow{3}{*}{10} & eficiente (>75\%) & 10 \\
\hline & & & admisible (50\% a 74\%) & 5 \\
\hline & & & deficiente $(<25 \%)$ & 1 \\
\hline
\end{tabular}

Fuente: Elaboración propia, 2018.

5. La seguridad vial. Tiene una valoración de 10/100 al interior del conjunto del inventario. Condicionantes claves que aumentan o disminuyen porcentajes de siniestros: i) semaforización, ii) velocidades máximas y iii) la calidad visual (Tabla 5 ).

\section{Tabla 5}

Valores y variables del parámetro seguridad

\begin{tabular}{|c|c|c|c|c|}
\hline PARÁMETRO & VARIABLES & ponderación & INDICADORES & V \\
\hline \multirow{7}{*}{$\begin{array}{c}\text { SEGURIDAD } \\
10 \% \\
\text { > a } 10 \text { Alta } \\
\text { e } 10 \text { a } 5 \text { Media } \\
\text { e } 5 \text { a } 0 \text { baja }\end{array}$} & & & eficiente (>75\%) & 2 \\
\hline & Semaforización & 2 & admisible (50\% a $74 \%$ ) & 1 \\
\hline & & & deficiente $(<25 \%)$ & 0.1 \\
\hline & & & $<80$ & 1 \\
\hline & Velocidades máximas & 6 & $>60$ & 3 \\
\hline & & & $>30$ & 6 \\
\hline & Legibilidad & 2 & si & 2 \\
\hline
\end{tabular}

Fuente: Elaboración propia, 2018. 
6. Parámetro paisajístico. Permite reconocer rangos de referencialidad de paisaje urbano, determinado la presencia de i) elementos naturales, ii) centralidades, nodos, hitos e iii) intersecciones y particularidades. Tiene una valoración de 10/100 al interior del conjunto del inventario (Tabla 6).

\section{Tabla 6}

Valores y variables del parámetro potencial paisajístico

\begin{tabular}{|c|c|c|c|c|}
\hline PARÁMETRO & VARIABLES & Ponderación & INDICADORES & $\mathbf{v}$ \\
\hline \multirow{9}{*}{$\begin{array}{c}\text { POTENCIAL } \\
\text { PAISAJÍSTICO } \\
10 \% \\
>4 \text { Alta } \\
\text { e } 4 \text { a } 2 \text { Media } \\
\text { < } 1 \text { baja }\end{array}$} & & & Alto & 5 \\
\hline & Presencia natural & 5 & Medio & 2 \\
\hline & & & Bajo & 0 \\
\hline & & & Alto (>40\%) & 2 \\
\hline & Centralidades/nodos/hitos & 2 & Medio (20\% a 39\%) & 1.5 \\
\hline & & & Bajo (<20\%) & 0.1 \\
\hline & & & Alto $(>40 \%)$ & 2 \\
\hline & Intersecciones & 2 & Medio (20\% a $39 \%)$ & 1.5 \\
\hline & & & Bajo (<20\%) & 0.1 \\
\hline
\end{tabular}

Fuente: Elaboración propia, 2018.

Los niveles de análisis se han realizado a partir de: i) datos de primera mano y fuentes secundarias: compilaciones, resúmenes y listados de referencias; ii) relevamientos fotográficos, peatonales. Siendo procesados y reelaborados por: i) Fichas y mapas temáticos mediante proyectos en sistemas de información geográfica y Statistical Package for the Social Sciences.

En relación con el trabajo con sistemas de información geográfica, al poder referenciarse en el espacio los datos en el total de la vía analizada, esto posibilita asignar rangos a los diferentes tramos, trasformando la variable cuantitativa en un rango de información cualitativa. A partir de esta valoración, se puede visualizar zonas con mayores grados de criticidad y potencialidades desde un enfoque general, comprendiendo la condición de este desde una forma particular y en el sistema que integra.

\section{Aplicación de la metodología: caso la plata}

\section{Sobre la ciudad de La Plata, desarrollo urbano, movilidad} e infraestructura

La ciudad de La Plata (Figura 3) capital de la provincia de Buenos Aires, forma parte de la denominada Región Metropolitana de Buenos Aires (RMBA). La Plata se constituye en la ciudad cabecera del Gran La Plata, cuenta con una población de 654.324 Habitantes, según censo 2010 y cuenta con una superficie de $942,23 \mathrm{~km}^{2}$.

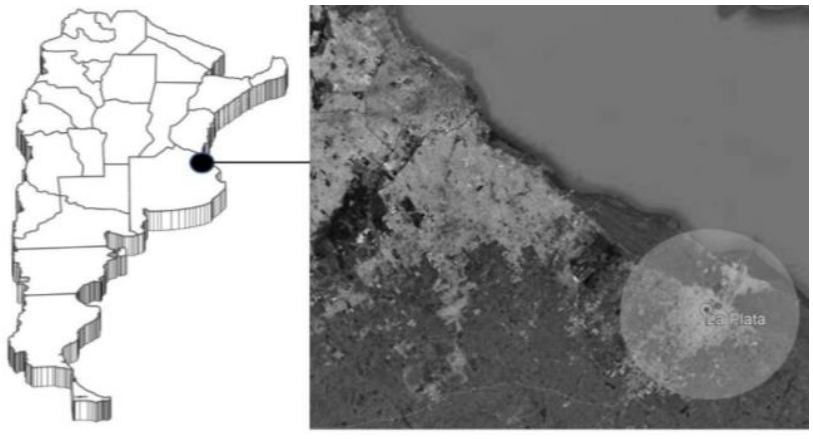

Figura 3. Situación geográfica de La Plata. Fuente: a partir de Google Earth (2019).

Esta ciudad se erigió sobre una zona litoral estuárica, característica geomorfológica que delineó el rol de la región como nodo de conexión entre el país y el exterior. La presencia del puerto fue el punto clave del desarrollo económico que, junto al despliegue de las infraestructuras de comunicación tanto hacia la capital federal como hacia el interior de las provincias, posibilitaron su consolidación. Sumado a esta actividad portuaria, la localización de las autoridades gubernamentales provinciales, junto con su administración municipal y la presencia de la Universidad Nacional; la posicionan como un nodo atractor y es lo que 
explica que la "conjunción del entorno administrativo instaurado en la ciudad con la radicación de universitarios sean dos factores de alto impacto sobre el desarrollo local" (Lódola y Brigo, 2011, p. 4).

La ciudad, por su población y actividades, integra el grupo de ciudades más importantes del país junto con el Área Metropolitana de Buenos Aires (AMBA), el Gran Rosario, el Gran Córdoba, el Gran Mendoza, el Gran Posadas y el Gran Tucumán. A su vez, comparte rasgos similares al conjunto, en relación con el desarrollo urbano y la movilidad. Estas regiones:

[R]egistran un proceso de expansión urbana que varía desde un $13 \%$ para el AMBA hasta un $34,5 \%$ a $65,3 \%$ para el resto de los aglomerados, mientras que en el caso del Gran La Plata la expansión asciende a un $114.5 \%$. Estas nuevas áreas urbanas, de bajísima densidad de habitantes (entre 6 y 20 habs./ha) crecen con una alta dependencia del automóvil y una pobre oferta de transporte público, como evidencia del desacoplamiento del desarrollo urbano y la movilidad de pasajeros. (Aón et al., 2017)

Particularmente La Plata presenta, por un lado, un área central o casco fundacional donde residen el 33\% del total de población de la ciudad y, por otro, cuatro zonas periféricas en constante crecimiento (Figura 4) que se diferencian principalmente por su configuración y perfil socioeconómico. Al respecto, la zona norte presenta un $26 \%$ de población, la oeste, $14 \%$ mientras que la zona este $16 \%$ y la zona sur $12 \%$, estas dos últimas son mayormente de carácter agropecuario.

El desarrollo urbano en los últimos años en el casco presenta tendencias de densificación y concentración de actividades que, combinado con la ausencia de planificación, resulta crítica en relación con la falta de adaptación de los servicios y equipamientos urbanos a los nuevos niveles de población. Al mismo tiempo, en las periferias se pueden reconocer tendencias de expansión residencial continuadas y creación de nuevas centralidades, que se estructuran sobre vías que conforman la red regional. Estas vías concentran la mayor parte del comercio y el tránsito interurbano liviano y público del Gran La Plata.
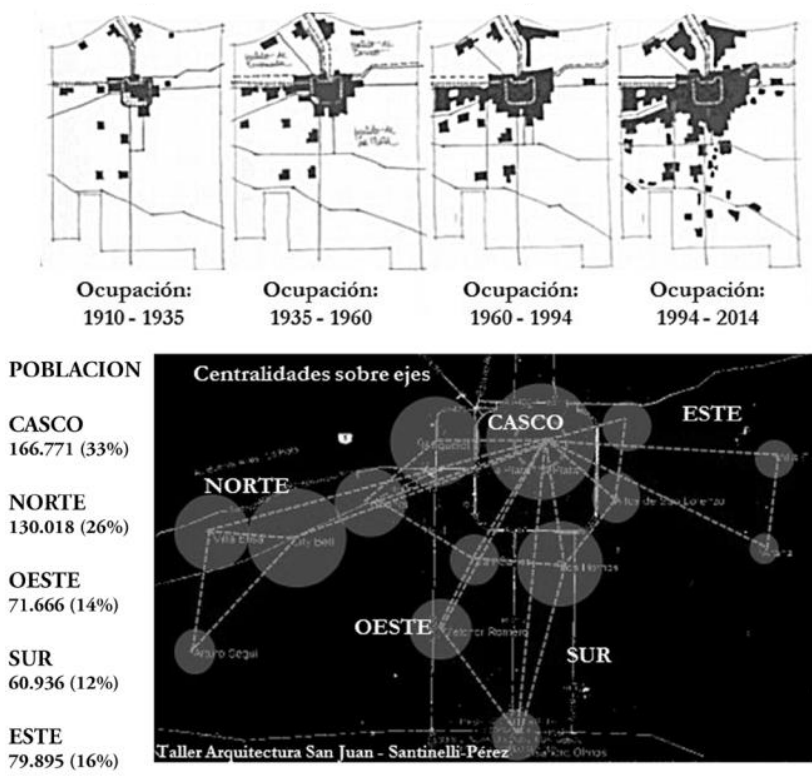

Figura 4. Desarrollo urbano y zonas de la plata. Fuente: Aón, López, Giglio, Freaza y Cola, 2019.

En este contexto, la infraestructura de la ciudad se organiza por grupo de redes vinculantes a nivel urbano y el regional (Figura 5). El nivel urbano (Artículo 124 de la Ordenanza 10703 Código de Ordenamiento Urbano de La Plata) se configura por una trama de calles y avenidas que posibilitan la vinculación entre los distintos espacios y las áreas centrales, así como también con las redes interurbanas regionales, metropolitanas y nacionales.

En este sentido, las redes interurbanas regionales se componen por vías cuya jerarquía confieren funcionalidad y entidad regional al área de influencia de La Plata quedando, junto con la red anterior, afectada al sistema de accesibilidad externo.

En relación con la movilidad, entre 1993-2003 el sistema público colectivo automotor de pasajeros perdió un $34 \%$ de los viajes diarios, la mayoría de los cuales fue absorbido por el automóvil particular (Aón et al., 2004). Por otro lado:

[D]e acuerdo a datos de la encuesta de movilidad 1993-2013, se registra que un $80 \%$ de la nueva demanda de viajes con destino a las áreas centrales, proviene de distintos sectores de la periferia de la ciudad. Esta situación, acorde al modelo de crecimiento centralizado de los usos no residenciales, explica en parte el incremento de autos circulando y la congestión cotidiana, y pone en evidencia un crítico desacople entre el 
desarrollo urbano y las prácticas urbanas de movilidad de los pasajeros. (Aón et al., 2017)

Particularmente, según la encuesta de movilidad 20032013, mientras en el casco tiende a un uso equitativito de medios de transporte, en las restantes zonas las diferencias se acentúan. En la zona Norte y Oeste la tendencia muestra mayor uso de medios no masivos motorizado (auto, moto). Mientras que en las otras dos zonas concentra mayor porcentaje los viajes en medios masivos motorizados (colectivo). Estos perfiles se explican, entre otros factores, a la falta de oferta espacial adecuada a las diferentes movilidades, como así también frente a las medidas implementadas a nivel municipal que solo atienen a cuestiones puntuales de corto plazo, como mejoramiento de pavimentación, ampliación de la red, instalación de equipamiento, lejos del planteamiento de instrumentos integrales. De ahí que, como expone el Observatorio de Movilidad de La Plata (2018) congestionamiento, niveles de ruido - contaminación niveles de accidentes y gasto del tiempo/viaje son las cuestiones actualmente más críticas, sobre todo en las vías que ofician de acceso al casco de la ciudad (López, 2016, p.176).

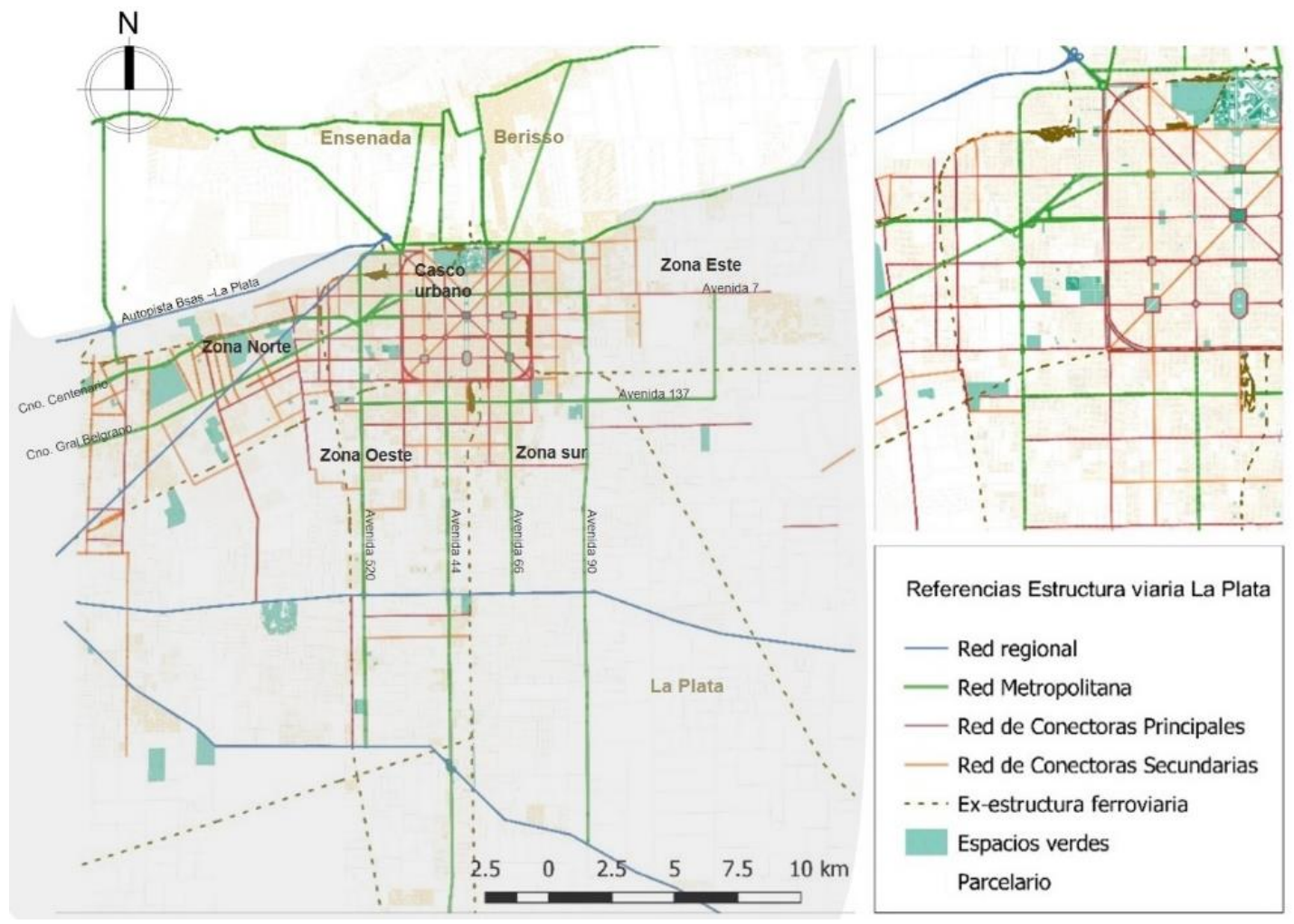

Figura 5. Sistema De Red Vial, Clasificación La Plata. Fuente: Elaboración propia, 2018. 


\section{Nivel superior de análisis: la infraestructura viaria y sus contextos}

Se detallan de forma sintética los resultados obtenidos del primer nivel de análisis y sus dos aproximaciones: histórica y urbano-territorial, dando cuenta lo importante de analizar la red viaria en sus contextos.

Aproximación histórica. La forma de crecimiento de la ciudad de La Plata evidencia evoluciones de la estructura espacial de la movilidad urbana que puede ser analizada según tres períodos históricos vinculados con los diferentes modelos técnico-económicos (Pérez, 2005). La ciudad, fundada en 1882, según las teorías urbanísticas del siglo XIX y en el momento que el acero era barato, se constituía como factor clave del modelo y los medios de transportes ferroviarios, emergentes en dicho desarrollo. En ese contexto, el ferrocarril como medio de vinculación interurbana y el tranvía como medio urbano vinculante, estructuraron la organización urbana (Ravella y Giacobbe, 2006).

La integración del sistema de transporte: red ferroviaria, red de caminos y sistema tranvía se articuló con el esquema de ciudad proyectado que se conformaba por tres componentes básicos estructurantes: casco urbano, zona de chacras y quintas y puerto (López, 2016, p.146).

El trazado de calles y avenidas se pensó de acuerdo con los criterios que se establecían en las ciudades europeas, entre ellas Paris y Barcelona. Las calles se constituyeron con un ancho de $18 \mathrm{~m}$ entre medianeras $\mathrm{y}$ veredas arboladas de $2,5 \mathrm{~m}$ de ancho. Las avenidas se estructuraron con anchos de $30 \mathrm{~m}$ entre medianeras $\mathrm{y}$ aceras arboladas de $4 \mathrm{~m}$, con diferentes variantes: i) con rambla (6,8 a 10,6 m variando la calzada y acera) diseños que pertenecían al casco y por fuera solo rambla verde; i) sin rambla; iii) con separador central. El arbolado fue implantado en el espacio público de manera diferente con el propósito de enriquecer la calidad paisajística urbana, promoviendo diversas sensaciones visuales (López, 2016, p.148).

La existencia previa del ferrocarril, que unía el puerto de Buenos Aires con la incipiente industria frigorífica localizada sobre el río de La Plata, determinó la creación del puerto de la ciudad y además generó la creación de núcleos poblacionales, vinculados con estaciones intermedias, entre ellas Villa Elisa, City Bell y Manuel B. Gonnet. Así se configura una estructura de tipo lineal fortalecida no solo por el trazado ferroviario, sino también por la pavimentación en 1907 del camino General Belgrano que fue, y todavía es, la principal vía de conexión entre la capital provincial y el primer cordón de conurbano.

A principios del siglo $\mathrm{XX}$ comienza a verificarse un cambio sustancial del modelo técnico-económico. El petróleo barato y el desarrollo de la industria automotriz, uno de sus emergentes, estimuló la aparición de los medios móviles de transporte: el automóvil y los modos públicos. En ese contexto, y por presión de los productores de automotores, se promueve la construcción de redes viarias que permitieran el desarrollo del transporte automotor, en detrimento del tranvía que será desactivado en la década de 1960 (Ravella, 2006). Las nuevas vías pavimentadas que vinculaban la ciudad con la capital del país y el resto de las ciudades provinciales sentaron las bases para el inicio de un modelo urbano automotor.

A partir de 1970, con la emergencia del nuevo modelo económico cuyos factores claves son el conocimiento y la informática, se consolida el neoliberalismo que provoca la aparición de nuevas formas consumo, el incremento de especulación inmobiliaria, la aparición de urbanizaciones cerradas, el crecimiento de los núcleos poblacionales periféricos y el desarrollo de asentamientos precarios, aumentando las diferencias espaciales y sociales. (Ravella, 2006; Frediani, 2010; Aón, 2012)

En la Figura 6 se presentan los rasgos principales de la evolución de la ciudad relacionadas con el desarrollo de la movilidad. En los años posteriores a su fundación, el casco urbano expresaba la condición de ciudad planificada adquiriendo las mejores innovaciones de la época y el sistema de movilidad (tren, tranvía y trolebús) otorgaba áreas con buenas condiciones de accesibilidad e integración, tanto interna como regional. Las avenidas y las plazas se constituían en símbolos urbanos, representando un modelo que hoy se denominaría sustentable. A partir de 1930, se evidencia que el crecimiento urbano se estructura a partir del desarrollo de ejes viarios y la conformación de centros y subcentros asociados. El desmantelamiento paulatino del sistema tranviario y ferroviario, así como también el deterioro del 
transporte público automotor y el crecimiento geométrico, modificaron drásticamente la funcionalidad inicial de calles y avenidas, provocando congestionamientos y obstrucción por el estacionamiento automotor, modificando drásticamente las condiciones de conexión y accesibilidad de diversas áreas de la ciudad.

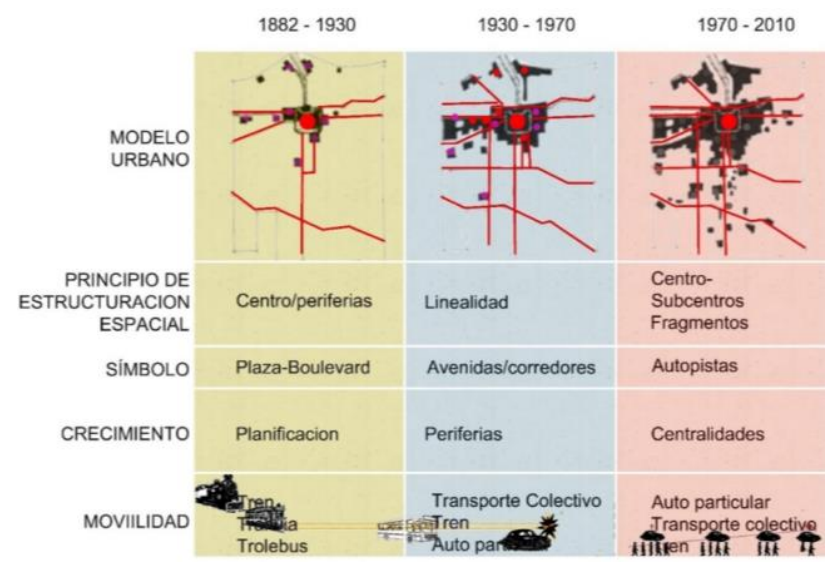

Figura 6. Evolución del modelo urbano, los principios de estructuración espacial, el crecimiento y movilidad de La Plata. Fuente: elaboración propia en base a datos Instituto Nacional de Estadística y Censos de la República Argentina INDEC, 2010.

Aproximación urbana - territorial: zonas urbanas y el sistema de red viario. El análisis previamente esbozado, permitió visibilizar los diferentes grados de desarrollo vinculados al sistema de comunicación, haciendo aún más evidente la necesidad de profundizar en las características sociales (población, hogares, vivienda, patrón de movilidad) y espaciales (barrios, sistema viario, hidrología, tejido, usos de suelo) particulares de cada zona. Cabe aclarar que para los objetivos de esta comunicación se seleccionó únicamente la zona Norte de las cuatro zonas periféricas para poder ejemplificar, destacar y detallar en profundidad los resultados del análisis urbano-territorial contextualizado a la red viaria y así poder continuar con el análisis.

Antes de exponer los resultados, interesa a modo de síntesis comparar entre el casco fundacional y las 4 zonas de crecimiento el grado de atractividad según sus usos. En el casco, se concentra el mayor porcentaje de actividades, comercios y servicios de la ciudad, concentrando el $45 \%$ de equipamientos de educación, el $30 \%$ de los equipamientos de salud, el $77 \%$ del equipamiento administrativo, el $56 \%$ del comercio y el $48 \%$ del espacio público total, quedando las periferias disminuidas en actividades complementarias a la vivienda. (Observatorio de Movilidad Gran La Plata, 2017). Mientras que, la composición de actividades residenciales y no residenciales fuera del casco, varían acorde a la antigüedad de la ocupación y conformación de las áreas y sus condiciones ambientales y sociales.

El área norte, tiene un $25 \%$ de equipamientos de educación, el $25 \%$ de los equipamientos de salud, el $20 \%$ del comercio y el $32 \%$ del espacio público total. Mientras que el área oeste, este y sur presentan similares porcentajes de equipamiento educativo (10\%), equipamiento de salud (10\%) y comercio (entre $6 \%$ y $10 \%$ ), destacándose la poca presencia de espacio verde.

A pesar de las diferencias de usos y actividades todas las zonas presentan una oferta espacial del viario que privilegia el espacio para el auto particular. Áreas peatonales, calles compartidas, carriles segregados para el transporte público de pasajeros o las bici-sendas son inexistentes. $\mathrm{Y}$, teniendo en cuenta los datos de la encuesta ${ }^{4}$ 1993/2013 que determinó que el 60\% de la movilidad se producía a través del auto particular y transporte público, mientras que el $36 \%$ restante se realiza a pie, bici, moto; es claro que más de un $36 \%$ de los viajes no tienen las condiciones adecuadas para su recorrido, por tanto, el $60 \%$ que viaja en auto o transporte, lo realiza argumentando no tener las condiciones adecuadas para realizarlo en otros modos.

Zona Norte: Subsistema social. En dicha área residen 54.306 hogares ( $21 \%$ del total del partido), un $14 \%$ más de población respecto al año 2001 , de los cuales más del $60 \%$ están compuestos por más de 4 integrantes. Esta tendencia en la ciudad de La Plata refleja una demanda residencial que, frente a la ausencia de restricciones normativas a la expansión y la falta de un plan de usos de suelo, promueven el crecimiento de periferias mayormente residenciales.

En este contexto, siguiendo a Aón (2017), el aumento de demanda residencial de familias de 4 o más integrantes de distinto nivel socio económico, tanto por la búsqueda

${ }^{4}$ Encuesta de movilidad del Gran La Plata 1993-2014. Grupo ii. IIPAC. en el marco Proyecto PPID Laura Aón. 
de mejores condiciones de vida (no proporcionadas por el casco urbano) o por imposibilidad de acceder y mantener una vivienda tipológica en el área central acorde a las necesidades de este tipo de hogares; provoca aumento de viajes al centro y un patrón modal que se distribuye en $53,6 \%$ de viajes motorizados no masivos (46\% automóvil; $1,65 \%$ taxi y $4,57 \%$ moto), un $32,8 \%$ de viajes motorizados masivos $(32,8 \%$ transporte publico automotor y $2,29 \%$ tren) y $13,6 \%$ no motorizados $(4,94 \%$ bicicleta y $8,49 \%$ a pie) (Figura 7).
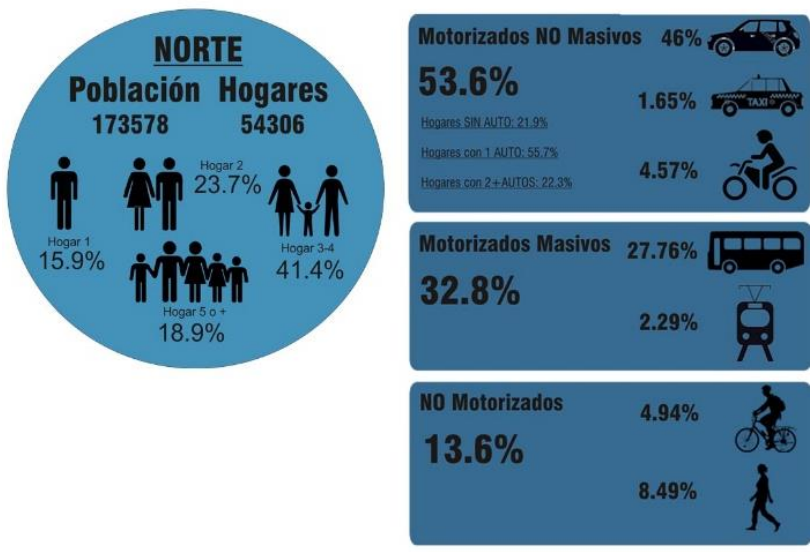

Figura 7. Zona Norte, distribución de hogares y patrón modal (Observatorio de Movilidad Gran La Plata, 2017). Fuente: Representación gráfica de María Luciana Giglio.

Zona Norte: Subsistema espacial. Conformada por 41 barrios que se agrupan en ocho delegaciones: Tolosa, Ringuelet, Hernández, Gorina, Gonnet, Villa Elisa, City Bell y Arturo Seguí. Las mismas se estructuran en torno a tres vías regionales metropolitanas, cada una se caracteriza según sus usos y alcances: el Camino Parque Centenario (CC) y las vías del ferrocarril General Roca (FFCC Gral. Roca) muestran una jerarquía de primer orden, concentrando la mayor parte del tránsito liviano y público, mientras que el Camino General Belgrano (CGB), conecta intrarregionalmente, concentrando tránsito interurbano del Gran La Plata (López, 2016, p.193) (Figura 8).

Los Caminos Centenario y Gral. Belgrano, generan en la localidad gran movimiento entre barrios, teniendo un carácter de vías articuladoras. Las mismas son de fácil acceso por medio de las conectoras principales: Avenidas 19 y 25 , a las que se articulan mediante conectoras secundarias: calles 485, 489, 501, 511 y 514. Asimismo, cada delegación se estructura por un sistema de redes primarias y secundarias, que no siguen los criterios determinados del casco de la ciudad.

El tejido urbano se caracteriza por ser continuo de tipo lineal compacto en cercanía al casco urbano y difuso a medida que se aleja (11 viv/ha). En este sector el uso del suelo es de uso residencial (46\%) con grandes equipamientos (26\%) y presencia de subcentros que se organizan y estructuran sobre los ejes viales primarios, concentrando las actividades comerciales, gastronómicas y recreativas.

Según datos provinciales, el incremento sistemático de flujo de alta velocidad en la red regional y metropolitana se debe en parte al proceso de crecimiento residencial y comercial. 


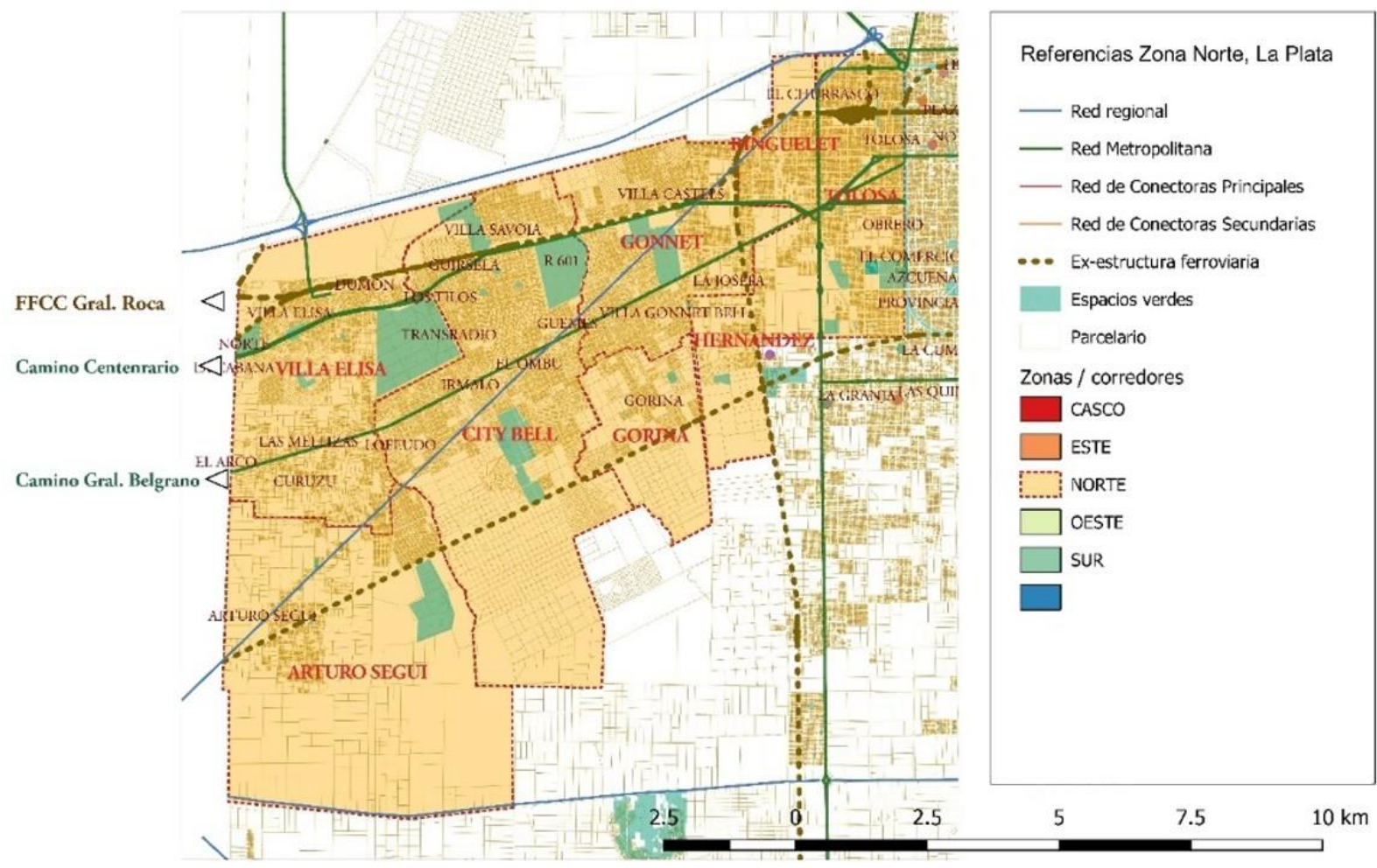

Figura 8. Zona Norte, delimitación de delegaciones y sistema viario. Fuente: Elaboración propia, 2018, a partir de Observatorio de Movilidad La Plata (2017).

Nivel de anclaje: Inventario vial: selección un corredor vial

De la estructura viaria de la zona norte, se seleccionó a modo de ejemplo una de las vías estructurantes del crecimiento de la zona: el Camino General Belgrano o Ruta Provincial 1 (Figura 9), por el mismo criterio aplicado en la aproximación urbano-territorial para poder profundizar mejor el análisis y exponer los resultados detallados del inventario y sus seis parámetros de diseño: 1) Vinculación socio espacial 2) espacial geométrico; 3) Integración funcional; 4) Habitabilidad; 5) seguridad y 6) potencial paisajístico.

1. Vinculación Socio Espacial. A partir de los datos registrados (Tabla 7) este parámetro expresa un alto grado de vinculación, ya que:

I. el tipo de vía según conteos y jerarquías representa una vía de acceso a la ciudad interurbana, metropolitana que conecta La Plata hasta
Avellaneda. Según Ordenanza Municipal de La Plata forma parte de la red regional al tener la función de vincular el partido de La Plata con otros centros urbanos y partidos adyacentes.

II. Dentro de esta zona el Camino General Belgrano o Ruta Provincial 1 tiene aproximadamente una longitud de 13,23 km entre avenida 32 y límite del Partido representando un rango medio.

III. Ofrece a nivel local conexión para el $26 \%$ de la población total del partido.

IV. Conecta de manera directa 8 delegaciones de las 19 que conforman a la ciudad es decir un $13 \%$ del total.

V. Atraviesa de manera directa 21 barrios consolidados con más de 161.407 habitantes, es decir $51 \%$ del total de barrios de la zona norte con un total de $93 \%$ de la población (173.251 habitantes). 


\section{Tabla 7}

Síntesis de los resultados

\begin{tabular}{|c|c|c|c|c|}
\hline PARÁMETRO & VARIABLES & Ponderación & Rangos & V \\
\hline \multirow{8}{*}{$\begin{array}{l}\text { VINCULACIÓN SOCIO } \\
\text { ESPACIAL }\end{array}$} & & \multirow{5}{*}{4} & Primer nivel urbana regional & 4 \\
\hline & & & segundo nivel urbana regional & 3 \\
\hline & $\begin{array}{l}\text { Tipo de vía según flujos conteos y jerarquía normada } \\
\text { Vía de acceso interurbana, metropolitana según la Ordenanza. }\end{array}$ & & primer nivel urbana & 3 \\
\hline & & & segundo nivel urbana & 2 \\
\hline & & & tercer nivel urbana & 1 \\
\hline & \multirow{3}{*}{$\begin{array}{l}\text { Longitud de la vía continua total en km } \\
\qquad 13,23 \mathrm{~km}\end{array}$} & \multirow{3}{*}{2} & alto $(>15 \mathrm{~km})$ & 3 \\
\hline & & & entre 15 km y 1 km & 2 \\
\hline & & & bajo $(<1$ km) & 1 \\
\hline \multirow{9}{*}{$\begin{array}{l}\text { > a } 15 \text { Alta } \\
\text { e } 14 \text { a } 5 \text { Media } \\
\text { e } 5 \text { a } 0 \text { baja }\end{array}$} & \multirow{3}{*}{$\begin{array}{l}\text { Población que ofrece conectividad \% del total de la ciudad } \\
\qquad 26 \% \text { del total de La Ciudad }\end{array}$} & \multirow{3}{*}{4 de 6} & Alto (>40\% población) & 6 \\
\hline & & & Medio (20\% a 39\% población) & 5 \\
\hline & & & Bajo (<20\% de población) & 2 \\
\hline & \multirow{3}{*}{$\begin{array}{l}\text { Nro. de delegaciones que conecta } \% \text { del total } \\
8 \text { delegaciones de } 19 \text { en total: } 13 \%\end{array}$} & \multirow{3}{*}{2 de 4} & Alto (> 40\% de las del.) & 4 \\
\hline & & & Medio ( $10 \%$ a $39 \%$ de las del.) & 2 \\
\hline & & & Bajo (<9\% de las del.) & 1 \\
\hline & \multirow{3}{*}{$\begin{array}{l}\text { Nro. barrios atravesados \% del total de esa zona } \\
\qquad 21 \text { de } 41 \text { barrios: } 51 \%\end{array}$} & \multirow{3}{*}{3} & Alto (>40\% de barrios) & 3 \\
\hline & & & Medio (20\% a $39 \%$ barrios) & 2 \\
\hline & & & Bajo (<20\% de barrios) & 1 \\
\hline
\end{tabular}

Fuente: Elaboración propia, 2019.

2. Condición geométrica-espacial. A partir de los datos registrados, este parámetro expresa un bajo nivel de flexibilidad según sus condiciones espaciales. Este corredor (Figura 9) fue pensado a principios del siglo $\mathrm{XX}$, para su ampliación posterior, restringiendo $5 \mathrm{~m}$ el uso de suelo de la edificación a cada lado de la vía. Esta restricción no fue respetada en la mayoría de su longitud. Actualmente la vía mantiene un ancho predominante de 7,9 $\mathrm{m}$ careciendo de veredas y bicisendas. Solo un tramo, de $7 \mathrm{~km}$ en el inicio de su trazado, tiene un ancho de $18 \mathrm{~m}$.
En su primer tramo se localizan grandes superficies comerciales, comercios diversos, viviendas de una planta localizadas en amplios terrenos arbolados, y un gran espacio de recreación (la República de los niños). En el resto del trayecto, actualmente se ha densificado con edificaciones de dos niveles, lo que ha incrementado la localización de población y, por lo tanto la movilidad hacia el centro urbano. En cuanto a la normativa, los indicadores del FOS/FOT/Altura y densidad que se establecen, rigen los mismos valores en todo el corredor: FOS 0.6/ FOT 1.2 y altura máxima de 3 niveles y densidad de 200 (Tabla 8). 

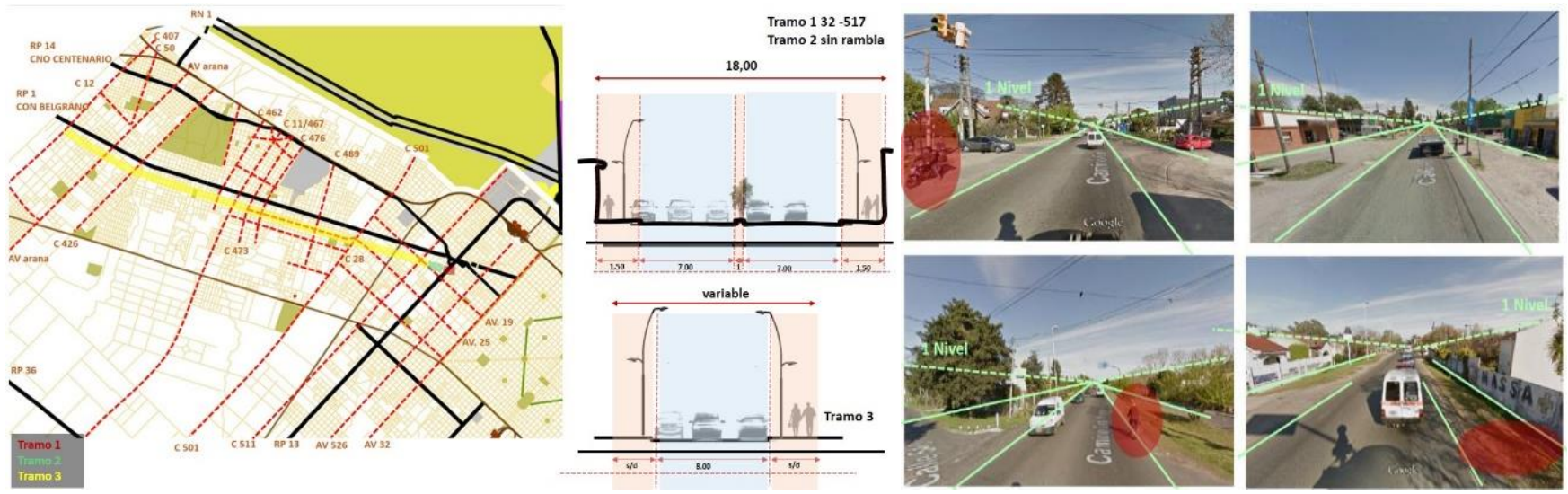

Figura 9. Secciones tipo del Camino General Belgrano. Fuente: Elaboración propia, 2019.

\section{Tabla 8}

Síntesis de los resultados

\begin{tabular}{|c|c|c|c|c|}
\hline PARÁMETRO & VARIABLES & Ponderación & Rangos & V \\
\hline \multirow{5}{*}{$\begin{array}{c}\text { ESPACIAL } \\
\text { GEOMÉTRICO } \\
15 \% \\
\text { > a } 15 \text { Alta } \\
\text { e } 14 \text { a } 5 \text { Media } \\
\text { < } 4 \text { baja }\end{array}$} & \multirow{3}{*}{$\begin{array}{l}\text { Actualmente la vía mantiene un ancho predominante de } \\
7,9 \mathrm{~m} \text { careciendo de veredas y bicis sendas }\end{array}$} & \multirow{3}{*}{1 de 10} & >20 m no consolidado & 10 \\
\hline & & & e/ 19 a 10 m no consolidado & 5 \\
\hline & & & $<10$ m consolidado & 1 \\
\hline & \multirow{3}{*}{ Alturas reales / normativa } & \multirow{3}{*}{3} & Perfil densificado +4 niveles & 1 \\
\hline & & & Perfil medio hasta 3 niveles & 3 \\
\hline Total: 4 baja & & & bajo 1 nivel & 5 \\
\hline
\end{tabular}

Fuente: Elaboración propia, 2019.

3. Integración funcional. A partir de los datos registrados (Tabla 9), este parámetro expresa un bajo grado de integración funcional entre la vía, su funcionalidad y el contexto que atraviesa debido a que:

I. El flujo vehicular que soporta 17.204 viajes diarios proyecta, de continuar el crecimiento demográfico, un incremento de un $16 \%$ de viajes para el 2020.

II. Las características del tráfico que se verifica en su recorrido, a diferencia de otros corredores, prevalece el auto particular y el transporte interurbano de pasajeros (Aón, 2017). Si bien el transporte de carga estuvo prohibido, actualmente el incremento de establecimientos que lo requieren ha incrementado su circulación.

III. Vincula 8 delegaciones de las 19 que conforman el partido.

IV. La gestión espacial del corredor es nula, el $100 \%$ se destina al medio motor.

V. Predomina uso comercial y residencial. 


\section{Tabla 9}

Síntesis de los resultados

\begin{tabular}{|c|c|c|c|c|}
\hline PARÁMETRO & VARIABLES & Ponderación & Rangos & V \\
\hline \multirow{10}{*}{$\begin{array}{c}\text { INTEGRACIÓN } \\
\text { FUNCIONAL } \\
20 \% \\
\text { > a } 15 \text { Alta } \\
\text { e } 14 \text { a } 5 \text { Media } \\
\text { e } 5 \text { a } 0 \text { baja }\end{array}$} & \multirow{3}{*}{$\begin{array}{c}17.204 \text { viajes } 2011 \text { proyección seg, pob. } 16 \% \text { más de } \\
\text { viajes } 2020\end{array}$} & \multirow{3}{*}{5} & Alto $(+20.000)$ & 5 \\
\hline & & & Medio (+4000) & 3 \\
\hline & & & Bajo (+2000) & 1 \\
\hline & \multirow{4}{*}{$\begin{array}{c}\text { Encuesta Origen Destino: } \\
\text { destaca por el mayor porcentaje de viajes utilizan el } \\
\text { automóvil particular } 41 \%\end{array}$} & \multirow{4}{*}{1 de 5} & Perfil mixto & 5 \\
\hline & & & Perfil público por sobre privado & 3 \\
\hline & & & Perfil privado por sobre público & 2 \\
\hline & & & privado & 1 \\
\hline & \multirow{3}{*}{$\begin{array}{l}\text { Numero de centralidades } \% \text { del total } \\
\qquad 8 \text { de } 19.13 \% \text { del total }\end{array}$} & \multirow{3}{*}{1 de 3} & Alto (> 40\%) & 3 \\
\hline & & & Medio (20\% a 39\%) & 2 \\
\hline & & & Bajo (<20\%) & 1 \\
\hline \multirow[t]{6}{*}{$10=$ media } & \multirow[t]{3}{*}{$\%$ de reparto espacial modal } & \multirow[t]{3}{*}{1 de 5} & $\begin{array}{l}\text { Eficiente } \% \mathrm{~m}^{2}[>40 \% \text { reparto } \\
\text { modal (bici-peatón-medios } \\
\text { motores)] }\end{array}$ & 5 \\
\hline & & & Admisible (> 40\% $\mathrm{m}^{2}$ motor) & 3 \\
\hline & & & Deficiente (<41\% $\mathrm{m}^{2}$ motor) & 1 \\
\hline & \multirow{3}{*}{ Perfil usos predominante } & \multirow{3}{*}{2} & Comercial & 2 \\
\hline & & & Mixto & 1.5 \\
\hline & & & Residencial & 1 \\
\hline
\end{tabular}

Fuente: Elaboración propia, 2019.

4. Parámetro de habitabilidad. El nivel de habitabilidad de la vía es inapropiado, con un total de 4 puntos sobre 25 debido a: i) mala calidad de la superficie, ii) inexistencia de aceras y bici-sendas, iii) equipamiento casi nulo en lo relacionado a la parada de buses y sus respectivos resguardos, v) la calidad sonora supera la admisible $y$, por último, iv) no tiene presencia del elemento vegetal tanto para su función de asoleamiento, como barrera sonora y de referencia (Tabla 10). 


\section{Tabla 10}

Síntesis de los resultados

\begin{tabular}{|c|c|c|c|c|}
\hline PARÁMETRO & VARIABLES & Ponderación & Rangos & v \\
\hline \multirow{9}{*}{$\begin{array}{l}\text { HABITABILIDAD } \\
25 \% \\
\text { > a } 10 \text { Alta } \\
\text { e } 9 \text { a } 5 \text { Media } \\
\text { e } 5 \text { a } 0 \text { baja }\end{array}$} & \multirow{3}{*}{$\begin{array}{l}\text { Superficies: variabilidad material } \\
\begin{array}{l}100 \% \text { asfalto, vereda mínima, césped o sin área provisoria } \\
\text { peatonal. }\end{array}\end{array}$} & \multirow{3}{*}{1 de 3} & eficiente (>75\%) & 3 \\
\hline & & & admisible (50\% a $74 \%)$ & 2 \\
\hline & & & deficiente $(<25 \%)$ & 1 \\
\hline & \multirow{3}{*}{$\begin{array}{l}\text { Superficies: estado } \\
\text { muy deteriorada con poca variabilidad. }\end{array}$} & \multirow{3}{*}{0.1 de 2} & eficiente (>75\%) & 2 \\
\hline & & & admisible (50\% a $74 \%)$ & 1 \\
\hline & & & deficiente $(<25 \%)$ & 0.1 \\
\hline & \multirow{3}{*}{$\begin{array}{l}\text { Valoración Cant. y estado de equipamiento } \\
\text { BAJO }\end{array}$} & \multirow{3}{*}{1} & eficiente $(>75 \%)$ & 2 \\
\hline & & & admisible ( $50 \%$ a $74 \%)$ & 1 \\
\hline & & & deficiente $(<25 \%)$ & 0.1 \\
\hline \multirow[t]{6}{*}{ 4,1 DE 25} & \multirow{3}{*}{$\begin{array}{c}\text { calidad sonora (dB/ inferencia TMDA) } \\
+ \text { de } 78 \mathrm{~dB}\end{array}$} & \multirow{3}{*}{1} & $<40 \mathrm{~dB}$ & 5 \\
\hline & & & e $41 \mathrm{~dB}$ a $65 \mathrm{~dB}$ & 3 \\
\hline & & & $>65 \mathrm{~dB}$ & 1 \\
\hline & \multirow{3}{*}{$\begin{array}{l}\% \text { presencia elemento vegetal (alta - media-baja) } \\
\text { BAJA }\end{array}$} & \multirow{3}{*}{1} & eficiente $(>75 \%)$ & 10 \\
\hline & & & admisible (50\% a $74 \%)$ & 5 \\
\hline & & & deficiente $(<25 \%)$ & 1 \\
\hline
\end{tabular}

Fuente: Elaboración propia, 2019.

5. Seguridad. A partir de los datos registrados (Tabla 11) este parámetro expresa un grado medio de seguridad de 5 puntos sobre 10 puntos, debido a que: i) el sistema de señalización y de semáforos es ineficiente, ii) la ausencia de elementos reguladores de velocidad y iii) la calidad que presenta en general el camino lo convierte en un ámbito obsoleto para la seguridad de cualquier grupo de población. En cuanto a la iv) legibilidad, no presenta elementos perturbadores.

Tabla 11

Síntesis de los resultados

\begin{tabular}{|c|c|c|c|c|}
\hline PARÁMETRO & VARIABLES & Ponderación & Rangos & $\mathbf{v}$ \\
\hline \multirow{4}{*}{$\begin{array}{c}\text { SEGURIDAD } \\
10 \% \\
>\text { a } 10 \mathrm{Alta} \\
\text { e } 10 \text { a } 5 \text { Media }\end{array}$} & \multirow{3}{*}{ Semaforización } & \multirow{3}{*}{1 de 2} & eficiente (>75\%) & 2 \\
\hline & & & admisible (50\% a74\%) & 1 \\
\hline & & & deficiente $(<25 \%)$ & 0.1 \\
\hline & \multirow{3}{*}{ Velocidades máximas } & \multirow{3}{*}{3 de 6} & $<80$ & 1 \\
\hline \multirow{3}{*}{$\begin{array}{c}\text { e } 5 \text { a } 0 \text { baja } \\
5=\text { media }\end{array}$} & & & $>60$ & 3 \\
\hline & & & $>30$ & 6 \\
\hline & Legibilidad & 2 & si & 2 \\
\hline
\end{tabular}

Fuente: Elaboración propia, 2019.

6. Potencial paisajístico. A partir de los datos registrados (Tabla 12), este parámetro expresa un grado medio de potencial de 3 puntos por sobre 10, debido a: i) una baja presencia de rasgos naturales. Se destaca la existencia de parques en las viviendas que se construyeron a partir de principios del siglo XX que se constituyen como elementos naturales que enmarcan la vía. Asimismo, ii) el factor flujo cotidiano y la función de vincular el partido de La Plata con otros atractivos de la zona, le confiere una potencialidad paisajística para posicionar una imagen urbana y de entidad regional. 


\section{Tabla 12}

Síntesis de los resultados

\begin{tabular}{|c|c|c|c|c|}
\hline PARÁMETRO & VARIABLES & Ponderación & Rangos & $\mathbf{V}$ \\
\hline \multirow{6}{*}{$\begin{array}{c}\text { POTENCIAL } \\
\text { PAISAJÍSTICO } \\
10 \% \\
\text { > a } 4 \text { Alta } \\
\text { e } 4 \text { a } 2 \text { Media } \\
\text { < } 1 \text { baja }\end{array}$} & \multirow{3}{*}{ Presencia natural } & \multirow{3}{*}{1 de 6} & Alto & 6 \\
\hline & & & Medio & 2 \\
\hline & & & Bajo & 0 \\
\hline & \multirow{3}{*}{$\begin{array}{l}\text { Centralidades/nodos/hitos } \\
\text { presenta atracciones destacadas }\end{array}$} & \multirow{3}{*}{1} & Alto (>40\%) & 2 \\
\hline & & & Medio (20\% a $39 \%)$ & 0.5 \\
\hline & & & Bajo (<20\%) & 0.1 \\
\hline \multirow{3}{*}{$3=$ media } & \multirow{3}{*}{ Intersecciones } & \multirow{3}{*}{1} & Alto (>40\%) & 2 \\
\hline & & & Medio (20\% a $39 \%)$ & 0.5 \\
\hline & & & Bajo (<20\%) & 0.1 \\
\hline
\end{tabular}

Fuente: Elaboración propia, 2019.

\section{Síntesis de los resultados.}

En la Tabla 13 se muestran los resultados y la ponderación junto al mapeo que resume rasgos y criticidades del análisis. A partir de los datos registrados el inventario demuestra que esta vía presenta un bajo nivel de calidad, funcionalidad y condiciona la movilidad y accesibilidad a la población ya que:

- Se destaca por un fuerte rol de conexión, pero las características físicas de la vía no responden a los flujos ni perfiles de movilidad que registra en la misma, provocando de esta manera problemas críticos de congestionamiento y accidentes. La falta de espacio entre autobuses, automóviles particulares, motos, bicicletas y peatones es la condicionante clave de esta criticidad.

- Este espacio viario fue y es un elemento estructurante en la organización espacial de la ciudad, tanto a nivel regional-urbano como a nivel barrial-local y el rol determinado desde que se concibió, según ordenanza, demuestra total irracionalidad frente a los cambios de crecimiento poblacional y usos. Retomando el nivel superior de análisis, esta vía fue concebida para conectar regionalmente a la ciudad, sin embargo, a pesar de su transformación, al momento de responder a otras demandas ha quedado totalmente obsoleta. Sus condiciones espaciales no funcionan ni colaboran con el sistema de red que constituye, ni tampoco colabora con los demás sistemas de redes.

- A su vez, por su diseño y estructura, la vía favorece a los medios motores e inhabilita al resto de los modos, posibilita altas velocidades y genera poca referencialidad según su calidad y señalización. Condicionar las formas de recorrer y usar el espacio público de la ciudad, representa una exclusión a los ciudadanos. 


\section{Tabla 13}

Síntesis de los resultados

\begin{tabular}{|c|c|}
\hline PARÁMETRO & VARIABLES \\
\hline \multirow{2}{*}{$\begin{array}{c}\text { POTENCIAL } \\
\text { PAISAJÍSTICO } \\
10 \%\end{array}$} & Tipo de Vía \\
\hline & Longitud de la Vía \\
\hline \multirow{2}{*}{$\begin{array}{c}\text { > a } 4 \text { Alta } \\
\text { e } 4 \text { a } 2 \text { Media } \\
\text { < } 1 \text { baja }\end{array}$} & Población que ofrece conectividad \\
\hline & Nro. De delegaciones que conecta \% \\
\hline $3=$ media & Nro. Barrios atravesados \% \\
\hline \multirow{2}{*}{$\begin{array}{c}\text { ESPACIAL } \\
\text { GEOMÉTRICO }\end{array}$} & Sección tipo \\
\hline & Alturas reales / normativa \\
\hline \multirow{3}{*}{$\begin{array}{l}\text { INTEGRACIÓN } \\
\text { FUNCIONAL }\end{array}$} & Tránsito Medio Diario Anual \\
\hline & Perfil movilidad \\
\hline & Número de centralidades \\
\hline \multirow[t]{2}{*}{$10=$ MEDIA } & $\%$ de reparto espacial modal \\
\hline & Perfil usos predominante \\
\hline \multirow{5}{*}{$\begin{array}{l}\text { HABITABILIDAD } \\
\text { e } 5 \text { A } 0 \text { Ваја }\end{array}$} & Superficies: variabilidad material \\
\hline & Superficies: estado \\
\hline & Valoración cant. Y estado de equipamiento \\
\hline & Calidad sonora (dB/ interferencia TMDA) \\
\hline & $\begin{array}{l}\% \text { presencia elemento vegetal (alta-media- } \\
\text { baja) }\end{array}$ \\
\hline \multirow{3}{*}{$\begin{array}{l}\text { SEGURIDAD } \\
\text { e } 10 \text { a } 5 \text { Media }\end{array}$} & Semaforización \\
\hline & Velocidades máximas \\
\hline & Legibilidad \\
\hline \multirow{2}{*}{$\begin{array}{c}\text { POTENCIAL } \\
\text { PAISAJÍSTICO }\end{array}$} & Presencia Natural \\
\hline & Centralidades/Nodos/Hitos \\
\hline e 4 a 2 Media & Intersecciones \\
\hline
\end{tabular}

Fuente: Elaboración propia, 2019.

\section{Conclusiones}

Las intervenciones realizadas en el sistema viario frente a las nuevas necesidades de la población toman como parámetros primordiales el flujo vehicular, la seguridad y la fluidez vehicular, lo que trae como resultado el aumento constante de: la dependencia de medios motores - más velocidad - Congestión - tiempo en viaje - Contaminación visual - sonora - atmosférica - espacial - disminución de la accesibilidad - medios de transporte postergados. Estas respuestas dejan entrever indirectamente una forma de concepción del elemento viario y modos de analizar los fenómenos que suceden en la ciudad, la movilidad y la accesibilidad. Desde la esfera política y normativa, no rige un interés por solucionar estos problemas de manera integral.

Desde este trabajo, en línea a nuevos avances teóricos, se valida un procedimiento de análisis del espacio viario, aplicando múltiples escalas y utilizando un sistema de variables integrales, con el fin de visualizar problemáticas y características en permanente transformación asociadas al sistema de las redes viarias. En este sentido, se valida el análisis que incluye la historicidad, ya que es fundamental para cualquier análisis urbano entender esas trasformaciones $y$, particularmente en relación con el sistema viario, permite resignificar su rol estructural en la configuración del territorio.

Asimismo, entender que la ciudad y sus sistemas componentes están en constante cambio, permite pensar medidas flexibles para solucionar problemáticas puntuales.

Por otro lado, se valida lo fundamental de analizar el ámbito territorial específico que se vincula con el viario. A partir de vincular dimensiones básicas de la configuración (sistema natural y sistema construido), de su organización (aspectos demográficos, actividad económica, usos) y la gestión urbana, todo esto coadyuva a dimensionar no solo necesidades de movilidad, velocidad; sino que permite entender el comportamiento de la red como sistema vinculante a actividades, tramas y grupos sociales totalmente diferentes.

Con relación al nivel de análisis del viario por medio del sistema de parámetros, queda validado el supuesto de que la estructura y diseño condicionan formas de la movilidad, admitiendo unos modos e impidiendo otros, posibilitando velocidades y generando referencialidad según su calidad y señalización. Al mismo tiempo que condicionan formas de recorrer y usar el espacio público de la ciudad y con ello, condicionan la inclusión de los grupos sociales a partir de la posibilidad de estos grupos de acceder a determinados modos de transporte.

La metodología y el inventario posibilitaron construir una visión totalizadora respecto a la realidad observada y asimismo, problematizar a cada parámetro y variable; lo cual contribuye a determinar: i) los aspectos que incrementan la desvinculación entre la red viaria, su función y el contexto espacial y social que atraviesan; ii) Los elementos que disminuyen/aumentan su 
acondicionamiento; iii) Cuáles parámetros son clave para guiar su diseño.

En el caso analizado se reconoce que el aspecto que incrementa la desvinculación de la vía está determinado, por un lado, por cuestiones técnico-políticas a partir de la aplicación de la ordenanza y, por otro lado, por estar asociado históricamente a un rol que, frente a los cambios de crecimiento poblacional, usos y movilidad, ha quedado totalmente obsoleto. Como consecuencia, sus condiciones no funcionan o colaboran ni con el sistema ciudad, ni con el sistema de red que constituye, así como tampoco colabora con los demás sistemas de redes.
Por último, se evidencia que la sección tipo, el perfil de movilidad dominante, la variabilidad y el estado de las superficies, la calidad sonora, la presencia vegetal; son variables clave en la disminución o aumento de su acondicionamiento. Por todo esto, los parámetros determinantes para guiar su diseño se corresponden a la habitabilidad, y al diseño espacial geométrico.

El articulo revaloriza diferentes enfoques necesarios para interpretar al espacio de la movilidad pero, por sobre todo, visibiliza el aporte de nuevas formas de análisis para el diseño urbano, abriendo las puertas a la investigación proyectual [R]

\section{Referencias}

UTDT -CIPUV Centro de Investigación de Políticas Urbanas y de Vivienda (2013). Atlas de Crecimiento Urbano. Disponible en http://atlasurbano.herokuapp.com/\#/

Aón, L. (2004) Evaluación de políticas de uso de suelo y transporte en el partido de La Plata- Informe Final. Beca de formación superior. Secretaria de Ciencia y Técnica, UI6B-IDEHAB- FAU-UNLP.

Aón, L. (2012). Transformaciones de la movilidad y de la accesibilidad urbana frente a los procesos de localización y re-localización residencial en el Partido de La Plata en el periodo 2001-2011. Proyectos de Investigación y Desarrollo. UNLP-SECYT.

Aón, L., Giglio, L. y Cola, C. (2017). Patrones modales de movilidad y desarrollo urbano no planificado en la ciudad de La Plata. Revista Transporte y Territorio, (17). Disponible en http://revistascientificas.filo.uba.ar/index.php/rtt/arti cle/view/3870

Aón, L., López, J., Freaza, N., Giglio, L. y Cola, C. (2017). Patrones de movilidad a nivel de hogar en el Gran La Plata. La Plata: Universidad Nacional de La Plata.

Appleyard, D., Lynch, K. \& Myer, J. (1964). The view from the road. Boston: M.I.T. Press.

Arroyo, J. (1992). Carreteras urbanas. Recomendaciones para su planeamiento y proyecto. Madrid: Ministerio de Obras Públicas y Transporte. Secretaria General Técnica.
Cullen, G. (1959). El paisaje urbano. Barcelona: Blume.

Crowe, S. (1960) Landscape of road. London: Architectural Press.

Dupuy, G. (1995). Les territoires de l'automobile. L'information géographique, 61(1), 41.

Fariña, J. (2012). Colin Buchanan, Traffic in Towns. Urbanismo, Territorio y Paisaje. [Fecha de consulta: 01-02-2015]. Disponible en http://elblogdefarina.blogspot.com.ar/

Freaza, N., Giglio, L. y Aón, L. (2017). Pérdida de complejidad en la ciudad compacta del mercado inmobiliario. Diez años de crecimiento del parque habitacional y desarrollo inmobiliario en área central de La Plata. Geograficando, 13(2), e028. https://doi.org/10.24215/2346898Xe028

Frediani, J. (2010). Lógicas y tendencias de la expansión residencial en áreas periurbanas. El partido de La Plata, Buenos Aires, Argentina, entre 1990 y 2010. La Plata: Universidad Nacional de La Plata. Disponible en http://sedici.unlp.edu.ar/handle/10915/3203

Halprin, L. (1966). Freeways. Editor: Reinhold

Herce, M (2009). Sobre la movilidad en la ciudad: propuestas para recuperar un derecho ciudadano. Barcelona: Reverté. 
Janoschka, M. (2002). El nuevo modelo de la ciudad latinoamericana: fragmentación y privatización. EURE, (28)85. https://doi.org/10.4067/s025071612002008500002

Jellicoe, G. (1966) Studies In Landscape Design. Oxford University Press.

Leva, G. (2012). Indicadores de calidad de vida urbana. Teoría y metodología. Buenos Aires: Universidad Nacional de Quilmes. Disponible en http://hm.unq.edu.ar/archivos hm/GL ICVU.pdf

Lódola, A y Brigo, R (2011). Diagnóstico Socioeconómico de La Plata y sus Centros Comunales. Serie Documentos de Trabajo. Departamento de Economía Facultad de Ciencias Económicas: Universidad Nacional de La Plata. Disponible en http://www.depeco.econo.unlp.edu.ar/wp/wpcontent/uploads/2017/05/doc87.pdf

López, M. J. (2016). Red viaria, ciudad y paisaje. Aproximación teórica metodológica para su diseño (Tesis doctoral). Universidad Nacional de La Plata. Disponible

en http://sedici.unlp.edu.ar/handle/10915/63160

Lynch, K. (1960). La imagen de la ciudad. Barcelona: Gustavo Gili.
Ministerio de Planificación Federal, Inversión Pública y Servicios (2011). Avance II: Argentina Urbana. Buenos Aires: Ministerio de Planificación Federal, Inversión Pública y Servicios.

NACTO (2013). Urban Street Design Guide. Island Press.

Naredo, J. y Rueda, S. (1996). La construcción de la ciudad sostenible: fundamentos. Madrid: Biblioteca Ciudades para un Futuro Más Sostenible. Disponible en http://habitat.aq.upm.es/cs/p2/lista.html.

Observatorio de Movilidad Urbana Gran La Plata. (2017). Tomo 1: La Plata. Instituto de Investigación y Políticas del Ambiente Construido, Facultad de Arquitectura y Urbanismo, Universidad Nacional de La Plata.

Pozueta Echavarri, J. (2014). Nuevos criterios para el diseño y clasificación del viario urbano. Carreteras: Revista técnica de la Asociación Española de la Carretera, (193), 33-55.

Ravella, O. (2000). El transporte como estructurador urbano. Una aproximación antropológica. FAU. UNLP

Samaja, J. (1996). El Proceso de la Ciencia, una breve introducción a la investigación científica. Buenos Aires: Secretaría de Investigación y Postgrado, FADU, UBA

Soria y Puig, A. (1999). Cerdá: the five bases of the general theory of urbanization. Madrid: Electa. 